\title{
Quantum information approach to electronic equilibria: molecular fragments and non-equilibrium thermodynamic description
}

\author{
Roman F. Nalewajski
}

Received: 7 November 2013 / Accepted: 10 April 2014 / Published online: 9 May 2014

C The Author(s) 2014. This article is published with open access at Springerlink.com

\begin{abstract}
The quantum-generalized Information Theory is applied to explore molecular equilibrium states by using the resultant information content of electronic states, determind by the classical (probability based) measures and their non-classical (phase/current related) complements, in the extremum entropy/information principles. The "vertical" (probability-constrained) entropic rules are investigated within the familiar Levy and Harriman-Zumbach-Maschke constructions of Density Functional Theory. A close parallelism between the vertical maximum-entropy and minimumenergy principles in quantum mechanics and their thermodynamic analogs is emphasized and a relation between the probability and phase distributions in the "horizontal" (probability-unconstrained) phase-equilibria is examined. These solutions are shown to involve the spatial phase contribution related to the system electron density. The complete specification of the equilibrium states of molecular/promolecular fragments, including the subsystem density and the equilibrium phase of the system as a whole, is advocated and illustrated for bonded hydrogens in $\mathrm{H}_{2}$. Elements of the non-equilibrium thermodynamic description of molecular systems are formulated. They recognize the independent probability and phase state parameters, the associated currents, and their contributions to the quantum entropy density and its current. The phase and entropy continuity equations are explored and the local sources of these quantities are identified.
\end{abstract}

Keywords Bonded molecular fragments · Classical/non-classical information terms · Entropy/phase continuity · Horizontal/vertical equilibria · Information principles $\cdot$ Molecular equilibria

R. F. Nalewajski $(\bowtie)$

Department of Theoretical Chemistry, Jagiellonian University, R. Ingardena 3,

30-060 Kraków, Poland

e-mail: nalewajs@chemia.uj.edu.pl 


\section{Introduction}

The Information Theory (IT) [1-8] has been successfully applied to explore the electron distributions and chemical bonds in molecules, e.g., [9-15]. It has been recently argued [16-19] that both the electron density, or its shape factor-the probability distribution determined by the wave-function modulus, and the system current distribution, related to the gradient of the wave-function phase, ultimately contribute to the resultant information content of the molecular electronic state. The particle density reveals the classical information content, while the probability current generates its non-classical complement in the resultant quantum information measure.

Many classical problems of theoretical chemistry have been approached afresh using this IT approach, e.g., [11-13], and the entropic probes of the molecular electronic structure have provided attractive tools for describing the chemical-bond phenomenon in information terms. This IT perspective introduces into the theory of electronic structure a novel entropy-representation, which complements the familiar energy-representation of the molecular quantum mechanics. Such a dual treatment parallels that encountered in the ordinary thermodynamics. It establishes the equivalent energy and entropy/information principles for determining the system equilibrium states, provides a new, unifying perspective on molecular states, extends a variety of tools for probing chemical processes, and enriches the range of available descriptors of the bonding patterns in molecules [11-15]. The IT criteria identify the system equilibrium states, corresponding to extrema of the quantum entropy/information functionals [16-19]. It also increases our understanding of the classical (intuitive) chemical concepts, e.g., the identity of Atoms-in Molecules (AIM) [9,10,20], electron/bond localization [11-15,21-23], sources and measures of the bond-multiplicity ("order") and its covalent/ionic composition [11-13,24], etc. The IT treatment leads to the "stockholder" AIM of Hirshfeld [25], which can be derived from alternative global or local variational principles of IT. The non-additive Fisher information in the Atomic Orbital (AO) resolution [26] has been used as the Contra-Gradience (CG) criterion for localizing bonding regions in molecules [11-15], while the related information (kinetic energy) density in the Molecular Orbital (MO) resolution has been shown [11,21-23] to determine the vital ingredient of the Electron-Localization Function (ELF) [27-29]. Moreover, the phenomenological description of equilibria in molecular subsystems has been proposed [30-32], which formally resembles that developed in the ordinary thermodynamics [33].

In the present work we focus on quantum measures of the entropy/information content of the molecular electronic states, and particularly on the non-classical contributions due to the wave-function phase, or its gradient - the probability current density. We then discuss the vertical and horizontal extrema of the non-classical and resultant entropy/information functionals, for the constrained and unconstrained classical contributions due to the electron density/probability distribution, respectively. The use of the equilibrium phases of subsystems as descriptors or their molecular/promolecular origins will be advocated and the elements of the non-equilibrium (irreversible) thermodynamic description will be given. This development involves the two functional state-parameters of the particle spatial probability and phase/current distributions, the 
associated fluxes and affinities, as well as the resulting quantum entropy current and source, the vital ingredients of the entropy continuity equation.

The extremum principles of the quantum-generalized information measures, possibly constrained by the extra requirements of conserving some "geometric" (normalization) and/or physical constraints, ultimately determine the associated molecular equilibria. In the present analysis we shall first explore the vertical principles, for the fixed electron density. We shall demonstrate that these energy and entropy/information rules in quantum mechanics, for the conserved system entropy/information and energy, respectively, resemble the complementary energy and entropy principles of the ordinary thermodynamics. The horizontal (probability unconstrained) entropic rules will explore the relation between the equilibrium phase and the system electron distribution. The horizontal (phase) equilibrium will be shown to exhibit spatial phase related to the system electron density, and hence also a non-vanishing probability current related to the gradient of the electron distribution.

Throughout the article the following tensor notation is used: $A$ denotes a scalar quantity, $\boldsymbol{A}$ stands for the row- or column-vector, and $\mathbf{A}$ represents a square or rectangular matrix. The logarithm of the Shannon-type information measure is taken to an arbitrary but fixed base. In keeping with the custom in works on IT the logarithm taken to base $2, \log =\log _{2}$, corresponds to the information measured in bits (binary digits), while selecting $\log =\ln$ expresses the amount of information in nats (natural units): 1 nat $=1.44$ bits.

\section{Quantum measures of the local entropy/information content}

Consider the molecular electron density $\rho(\boldsymbol{r})=N p(\boldsymbol{r})$ and its shape (probability) distribution $p(\boldsymbol{r})=[A(\boldsymbol{r})]^{2}$, the square of the classical amplitude $A(\boldsymbol{r})$, where $N$ stands for the system overall number of electrons. Let us first examine the simplest case of a single electron in the (complex) variational state at the initial time $t_{0} \equiv 0$,

$$
\varphi(\boldsymbol{r})=R(\boldsymbol{r}) \exp [\mathrm{i} \phi(\boldsymbol{r})]=\varphi[R, \phi ; \boldsymbol{r}]
$$

Its modulus part, $R(\boldsymbol{r})$, represents the classical amplitude $A(\boldsymbol{r})$ of the (normalized) spatial probability distribution,

$$
p(\boldsymbol{r})=\varphi^{*}(\boldsymbol{r}) \varphi(\boldsymbol{r})=R(\boldsymbol{r})^{2}, \quad \int p(\boldsymbol{r}) d \boldsymbol{r}=1,
$$

while the gradient of its (spatial) phase component $\phi(\boldsymbol{r})$ generates the associated probability current density:

$\boldsymbol{j}(\boldsymbol{r})=\frac{\hbar}{2 m i}\left[\varphi^{*}(\boldsymbol{r}) \nabla \varphi(\boldsymbol{r})-\varphi(\boldsymbol{r}) \nabla \varphi^{*}(\boldsymbol{r})\right]=\frac{\hbar}{m} \operatorname{Im}\left[\varphi^{*}(\boldsymbol{r}) \nabla \varphi(\boldsymbol{r})\right]=\frac{\hbar p(\boldsymbol{r})}{m} \nabla \phi(\boldsymbol{r})$,

In the typical molecular scenario one envisages a single electron moving in an external potential $v(\boldsymbol{r})$ due to the fixed nuclei (Born-Oppenheimer approximation), say, in the diatomic molecule $\mathrm{A}-\mathrm{B}$, e.g., in $\mathrm{H}_{2}^{+}$, described by the Hamiltonian 


$$
\hat{\mathrm{H}}(\boldsymbol{r})=-\left(\hbar^{2} / 2 m\right) \nabla^{2}+v(\boldsymbol{r})
$$

Its eigensolutions $\left\{\varphi_{i}(\boldsymbol{r})\right\}$ are determined by the stationary Schrödinger equation (SE):

$$
\hat{\mathrm{H}}(\boldsymbol{r}) \varphi_{i}(\boldsymbol{r})=E_{i} \varphi_{i}(\boldsymbol{r}), \quad i=0,1,2 \ldots
$$

They correspond to the sharply specified energies $\left\{E_{i}\right\}$, eigenvalues of the Hamiltonian, with the lowest eigenvalue, for $i=0$, corresponding to the system ground state, and the stationary (time independent) probability distribution $p_{i}(\boldsymbol{r})=\left[R_{i}(\boldsymbol{r})\right]^{2}$. One also recalls that the non-degenerate eigenstates of the electronic Hamiltonian correspond to the vanishing spatial phase, $\phi_{i}(\boldsymbol{r})=0 \equiv \phi_{0}(\boldsymbol{r})$, i.e., $\varphi_{i}(\boldsymbol{r})=R_{i}(\boldsymbol{r})$, and hence also to the vanishing current $\boldsymbol{j}_{i}(\boldsymbol{r})=\boldsymbol{0}$. The two independent components $[R, \phi]$ or $[p, \boldsymbol{j}]$, of a general (complex) electronic state $\varphi(\boldsymbol{r})=\varphi[R, \phi ; \boldsymbol{r}]=\varphi[p, \boldsymbol{j} ; \boldsymbol{r}]$, thus provide the complete specification of the particle quantum state at $t_{0}$ and its resultant quantum entropy/information content [16-19].

When examining the dynamics of such quantum states one allows the time dependence of both these components in the full quantum state

$$
\varphi(\boldsymbol{r}, t)=R(\boldsymbol{r}, t) \exp [\mathrm{i} \phi(\boldsymbol{r}, t)] \equiv\langle\boldsymbol{r} \mid \varphi(t)\rangle .
$$

Its time evolution is then described by the time-dependent SE,

$$
\mathrm{i} \hbar \partial \varphi(\boldsymbol{r}, t) / \partial t=\hat{\mathrm{H}}(\boldsymbol{r}) \varphi(\boldsymbol{r}, t),
$$

which marks the stationary quantum action:

$$
A[\varphi(t)]=\langle\varphi(t)|\mathrm{i} \hbar \partial / \partial t-\hat{\mathrm{H}}| \varphi(t)\rangle
$$

One also recalls that the full stationary state of Eq. (5) exhibits the purely timedependent phase:

$$
\begin{aligned}
\varphi_{i}(\boldsymbol{r}, t) & =\left\langle\boldsymbol{r} \mid \varphi_{i}(t)\right\rangle \\
& =\varphi_{i}(\boldsymbol{r}) \exp \left[-\mathrm{i}\left(E_{i} / \hbar\right) t\right] \equiv R_{i}(\boldsymbol{r}) \exp \left[-\mathrm{i} \omega_{i} t\right] \equiv R_{i}(\boldsymbol{r}) \exp \left[\mathrm{i} \phi_{i}(t)\right],
\end{aligned}
$$

and hence the vanishing current of Eq. (3).

Both the probability distribution and its phase/current density ultimately contribute to the resultant information content of quantum states [16-19]. The non-classical entropy or information terms allow one to distinguish between quantum states exhibiting the same electron density but differing in patterns of their probability currents. Therefore, the wave-function modulus (amplitude of the particle density/probability function) and its phase (or phase-gradient, i.e., the current density) constitute two fundamental "degrees-of-freedom" in the complete quantum IT description of the system electronic states: $\varphi \Leftrightarrow(R, \phi) \Leftrightarrow(p, \boldsymbol{j})$. 
The SE (7) and its Hermitian conjugate give rise to the probability-continuity equation,

$$
\begin{aligned}
\partial p / \partial t & =-\nabla \cdot \boldsymbol{j} \quad \text { or } \\
d p / d t \equiv \dot{p} & =\sigma_{p}=\partial p / \partial t+\nabla \cdot \boldsymbol{j}=0,
\end{aligned}
$$

which expresses a local balance in the electron rate processes. This $(p, \boldsymbol{j})$-transformed SE shows that the local change in the probability density [1.h.s of Eq. (10)] is solely due to the probability outflow measured by the negative divergence of the probability current density [r.h.s. of Eq. (10)]. The vanishing total time derivative (the probability source) of Eq. (11), $\dot{p}=\sigma_{p}=0$, which expresses the time rate of change of the particle density in an infinitesimal "monitoring" volume element flowing with the particle, signifies the sourceless density/probability redistributions. Indeed, the molecular extensive parameter $\rho(\boldsymbol{r})$ can be neither produced nor destroyed. It also follows from the preceding equation that the probability (wave-function) norm remains conserved in time,

$$
\partial / \partial t\left[\int p(\boldsymbol{r}, t) d \boldsymbol{r}\right]=\partial / \partial t\left[\int \varphi^{*}(\boldsymbol{r}, t) \varphi(\boldsymbol{r}, t) d \boldsymbol{r}\right]=\int[\partial p(\boldsymbol{r}, t) / \partial t] d \boldsymbol{r}=0 .
$$

The probability current per particle, $(\boldsymbol{j} / p) \equiv \boldsymbol{V}$, measures the local velocity $\boldsymbol{V}$ of this density/probability "fluid", determined by the gradient of the phase part of the system wave-function:

$$
\begin{aligned}
\nabla \phi & =(m / \hbar)(\boldsymbol{j} / p)=(m / \hbar) \boldsymbol{V} \\
& =p^{-1} \operatorname{Im}\left[\varphi^{*} \nabla \varphi\right] .
\end{aligned}
$$

Consider the non-classical, phase/current-related complement [16-19],

$$
\begin{aligned}
S^{\text {nclass. }}[\varphi] & =\left\langle\varphi\left|\hat{\mathrm{S}}_{\varphi}\right| \varphi\right\rangle=-2 \int p(\boldsymbol{r})\left[\phi^{2}(\boldsymbol{r})\right]^{1 / 2} d \boldsymbol{r} \equiv-2 \int p(\boldsymbol{r})[\pi(\boldsymbol{r})]^{1 / 2} d \boldsymbol{r}=-2\langle|\phi|\rangle_{\varphi} \\
& \equiv S[p, \phi] \equiv \int p(\boldsymbol{r}) S^{n \text { class. }}(\boldsymbol{r}) d \boldsymbol{r} \\
\hat{\mathrm{S}}_{\phi}(\boldsymbol{r}) & =-2\left[\phi^{2}(\boldsymbol{r})\right]^{1 / 2}=-2|\phi(\boldsymbol{r})|=-2 \pi(\boldsymbol{r})^{1 / 2}=S_{\varphi}(\boldsymbol{r}),
\end{aligned}
$$

to the classical entropy density $S^{\text {class. }}(\boldsymbol{r}) \equiv S_{p}(\boldsymbol{r})$ of familiar Shannon functional in the classical, probability-based IT [3,4],

$$
\begin{array}{r}
S^{\text {class. }}[\varphi]=\left\langle\varphi\left|\hat{\mathrm{S}}_{p}\right| \varphi\right\rangle=-\int p(\boldsymbol{r}) \log p(\boldsymbol{r}) d \boldsymbol{r} \\
=\langle-\log p\rangle_{\varphi} \equiv S[p] \equiv \int p(\boldsymbol{r}) S^{\text {class. }}(\boldsymbol{r}) d \boldsymbol{r} \\
\hat{\mathrm{S}}_{p}(\boldsymbol{r})=-\log p(\boldsymbol{r})=S_{p}(\boldsymbol{r}) .
\end{array}
$$


The non-classical density-per-electron $S_{\varphi}(\boldsymbol{r})$ is proportional to the local magnitude of the phase function, $|\phi|=\left[\phi^{2}\right]^{1 / 2}$, the square root of the phase-density $\pi=\phi^{2}$, with the particle probability $p$ providing the local "weighting" factor in the associated average (global) functional, while the classical density $S^{\text {class. }}(\boldsymbol{r})$ is seen to be determined by the negative logarithm of the system probability density. Together these two components generate the overall, resultant Shannon measure of the quantum indeterminicity content due to both the probability and current distributions in the complex quantum state $\varphi[16-19]$ :

$$
\begin{aligned}
S[\varphi] & =S^{\text {class. }}[\varphi]+S^{\text {nclass. }}[\varphi]=\langle\varphi|\hat{\mathrm{S}}| \varphi\rangle \equiv \int p(\boldsymbol{r}) S(\boldsymbol{r}) d \boldsymbol{r} \equiv \int \mathscr{S}(\boldsymbol{r}) d \boldsymbol{r}, \\
\hat{\mathrm{S}}(\boldsymbol{r}) & =\hat{\mathrm{S}}_{p}(\boldsymbol{r})+\hat{\mathrm{S}}_{\varphi}(\boldsymbol{r})=S_{p}(\boldsymbol{r})+S_{\varphi}(\boldsymbol{r})=S(\boldsymbol{r}),
\end{aligned}
$$

where $\mathscr{S}(\boldsymbol{r})=p(\boldsymbol{r}) S(\boldsymbol{r})$ stands for overall density of the quantum entropy functional.

We also recall that the Fisher information for locality events [1,2], called the intrinsic accuracy, provides the complementary (gradient) measure of the classical information content in the quantum state $\varphi(r)$ :

$$
\begin{aligned}
I^{\text {class. }[\varphi]} & =\left\langle\varphi\left|\hat{\mathrm{I}}_{p}\right| \varphi\right\rangle=\int p(\boldsymbol{r})[\nabla \ln p(\boldsymbol{r})]^{2} d \boldsymbol{r}=\left\langle(\nabla \ln p)^{2}\right\rangle_{\varphi} \\
& =\int[\nabla p(\boldsymbol{r})]^{2} / p(\boldsymbol{r}) d \boldsymbol{r}=I[p]=4 \int[\nabla A(\boldsymbol{r})]^{2} d \boldsymbol{r} \equiv I[A], \\
\hat{\mathrm{I}}_{p}(\boldsymbol{r}) & =[\nabla \ln p(\boldsymbol{r})]^{2}=[\nabla p(\boldsymbol{r}) / p(\boldsymbol{r})]^{2}=I_{p}(\boldsymbol{r})=4[\nabla R(\boldsymbol{r})]^{2} .
\end{aligned}
$$

This classical-amplitude functional $I[A]$ is then naturally generalized into the domain of complex probability amplitudes (wave functions) of molecular quantum mechanics [26],

$$
I[\varphi]=4 \int|\nabla \varphi(\boldsymbol{r})|^{2} d \boldsymbol{r}=\int \mathscr{I}(\boldsymbol{r}) d \boldsymbol{r} \equiv \frac{8 m}{\hbar^{2}} T[\varphi]
$$

The quantum information functional is thus seen to be related to the expectation value of the electronic kinetic energy:

$$
T[\varphi] \equiv\langle\phi|\hat{\mathrm{T}}| \phi\rangle=-\frac{\hbar^{2}}{2 m} \int \phi^{*}(\boldsymbol{r}) \Delta \phi(\boldsymbol{r}) d \boldsymbol{r}=\frac{\hbar^{2}}{2 m} \int|\nabla \phi(\boldsymbol{r})|^{2} d \boldsymbol{r} .
$$

This kinetic energy functional consists of the classical, von Weizsäcker's [34] contribution,

$$
\begin{aligned}
T[p]= & \frac{\hbar^{2}}{8 m} \int \frac{[\nabla p(\boldsymbol{r})]^{2}}{p(\boldsymbol{r})} d \boldsymbol{r}=\frac{\hbar^{2}}{2 m} \int[\nabla R(\boldsymbol{r})]^{2} d \boldsymbol{r} \\
& =T^{\text {class. }}[\varphi]=\left\langle\varphi\left|T_{p}\right| \varphi\right\rangle, \\
T_{p}(\boldsymbol{r})= & \frac{\hbar^{2}}{8 m}[\nabla p(\boldsymbol{r}) / p(\boldsymbol{r})]^{2}=\frac{\hbar^{2}}{2 m}[\nabla R(\boldsymbol{r}) / R(\boldsymbol{r})]^{2},
\end{aligned}
$$


depending solely upon the electron probability density, and the non-classical, (phase/current)-related term

$$
\begin{aligned}
T[p, \boldsymbol{j}] & =\frac{m}{2} \int \frac{\boldsymbol{j}(\boldsymbol{r})^{2}}{p(\boldsymbol{r})} d \boldsymbol{r}=\frac{\hbar^{2}}{2 m} \int p(\boldsymbol{r})[\nabla \varphi(\boldsymbol{r})]^{2} d \boldsymbol{r} \\
& =T[p, \phi]=T^{\text {nclass. }}[\varphi]=\left\langle\varphi\left|T_{\varphi}\right| \varphi\right\rangle, T_{\varphi}(\boldsymbol{r})=\frac{\hbar^{2}}{2 m}[\nabla \varphi(\boldsymbol{r})]^{2} .
\end{aligned}
$$

This probability/phase separation gives a transparent partition of the overall kinetic energy functional:

$$
\begin{aligned}
T[\varphi] & =\langle\varphi|\hat{\mathrm{T}}| \varphi\rangle=T[p]+T[p, \phi]=T^{\text {class. }}[\varphi]+T^{\text {nclass. }}[\varphi] \\
& =\int p(\boldsymbol{r}) T(\boldsymbol{r}) d \boldsymbol{r}, \quad T(\boldsymbol{r})=T_{p}(\boldsymbol{r})+T_{\varphi}(\boldsymbol{r})
\end{aligned}
$$

Notice, however, that the effective (multiplicative, real) "operator" $T(\boldsymbol{r})$ measuring the density-per-electron of the probability functional $T[p]$, derived from equality of the quantum expectation values,

$$
\langle\varphi|\hat{\mathrm{T}}| \varphi\rangle=\langle\varphi|T| \varphi\rangle=\int T(\boldsymbol{r}) p(\boldsymbol{r}) d \boldsymbol{r}=T[p],
$$

differs from the true (differential) kinetic energy operator $\hat{\mathrm{T}}(\boldsymbol{r})=-\left(\hbar^{2} / 2 m\right) \nabla^{2}$.

A similar division applies to the resultant, quantum Fisher information:

$$
\begin{aligned}
I[\varphi] & =I^{\text {class. }}[\varphi]+I^{\text {nclass. }}[\varphi] \equiv \int p(\boldsymbol{r})\left[I^{\text {class. }}(\boldsymbol{r})+I^{\text {nclass. }}(\boldsymbol{r})\right] d \boldsymbol{r} \\
& =I[p]+4 \int p(\boldsymbol{r})[\nabla \phi(\boldsymbol{r})]^{2} d \boldsymbol{r} \equiv I[p]+I[p, \phi] \\
& =I[p]+4\left(\frac{m}{\hbar}\right)^{2} \int \frac{\boldsymbol{j}^{2}(\boldsymbol{r})}{p(\boldsymbol{r})} d \boldsymbol{r} \equiv I[p]+I[p, \boldsymbol{j}],
\end{aligned}
$$

where the relevant information densities-per-electron read:

$$
\begin{aligned}
I^{\text {class. }}(\boldsymbol{r}) & =[\nabla p(\boldsymbol{r}) / p(\boldsymbol{r})]^{2}=4[\nabla R(\boldsymbol{r})]^{2}, \\
I^{\text {nclass. }}(\boldsymbol{r}) & =4(\mathrm{~m} / \hbar)^{2}[\boldsymbol{j}(\boldsymbol{r}) / p(\boldsymbol{r})]^{2}=4[\nabla \phi(\boldsymbol{r})]^{2} .
\end{aligned}
$$

These two Fisher-information components can be thus expressed as the quantum mechanical expectation values of the related (multiplicative) "operators" (information densities):

$$
\begin{aligned}
& I^{\text {class. }}[\varphi]=\left\langle\varphi\left|I_{p}\right| \varphi\right\rangle, \quad I_{p}(\boldsymbol{r})=[\nabla p(\boldsymbol{r}) / p(\boldsymbol{r})]^{2}, \quad \text { and } \\
& I^{\text {nclass. }}[\varphi]=\left\langle\varphi\left|I_{\phi}\right| \varphi\right\rangle, \quad I_{\phi}(\boldsymbol{r})=4[\nabla \phi(\boldsymbol{r})]^{2} \text {, }
\end{aligned}
$$


giving the associated expression for the resultant quantum information measure:

$$
\begin{aligned}
I[\varphi] & =I[p]+I[p, \phi]=I^{\text {class. }}[\varphi]+I^{\text {nclass. }}[\varphi]=\langle\varphi|I| \varphi\rangle \\
& =\int p(\boldsymbol{r}) I(\boldsymbol{r}) d \boldsymbol{r}=\int \mathscr{I}(\boldsymbol{r}) d \boldsymbol{r}, \quad I(\boldsymbol{r})=I_{p}(\boldsymbol{r})+I_{\phi}(\boldsymbol{r})
\end{aligned}
$$

Both the classical and non-classical densities-per-electron of the complementary Shannon and Fisher measures of the quantum information content are mutually related via the common-type dependence [16-19]:

$$
\begin{gathered}
I^{\text {class. }}(\boldsymbol{r})=[\nabla \ln p(\boldsymbol{r})]^{2}=\left[\nabla S^{\text {class. }}(\boldsymbol{r})\right]^{2} \quad \text { and } \\
I^{\text {nclass. }}(\boldsymbol{r})=\left(\frac{2 m \boldsymbol{j}(\boldsymbol{r})}{\hbar p(\boldsymbol{r})}\right)^{2} \equiv\left[\nabla S^{\text {nclass. }}(\boldsymbol{r})\right]^{2} .
\end{gathered}
$$

In other words, the square of the gradient of the local Shannon probe in the state resultant quantum "indeterminicity" (disorder) generates the density of the corresponding Fisher measure of the state quantum "determinicity" (order).

To summarize, the system electron distribution, related to the wave-function modulus, reveals the probability (classical) aspect of the molecular information content [1-8], while the phase(current) facet of the molecular state gives rise to the specifically quantum (non-classical) entropy/information terms [16-19]. Together these two contributions allow one to monitor the full information content of the non-equilibrium or variational quantum states, providing the complete IT description of their evolution towards the final equilibrium.

\section{Vertical solutions and horizontal phase-equilibria}

As in ordinary thermodynamics, the lowest (stationary) equilibrium of the molecular ground state $\varphi_{0}$ alternatively results either from the minimum-energy principle, groundstate entropy constrained, or from complementary, ground-state energy constrained IT principles: of the maximum of the non-classical Shannon entropy or the minimum of the quantum Fisher information [17,19]. To illustrate this point we again refer to the illustrative example of a single particle described by the Hamiltonian of Eq. (4) in the trial state $\varphi(\boldsymbol{r})$ of Eq. (1).

Consider first the minimum principle of the expectation value of the system electronic energy in the modulus-constrained trial state $\varphi^{0}(\boldsymbol{r})=R_{0}(\boldsymbol{r}) \exp [\mathrm{i} \phi(\boldsymbol{r})]$, where $R_{0}(\boldsymbol{r})=\left[p_{0}(\boldsymbol{r})\right]^{1 / 2}$,

$$
\begin{aligned}
E_{v}\left[\varphi^{0}\right]=\left\langle\phi^{0}|\hat{\mathrm{H}}| \phi^{0}\right\rangle= & \left(\hbar^{2} / 2 m\right) \int\left[\left(\nabla R_{0}\right)^{2}+R_{0}^{2}(\nabla \varphi)^{2}\right] d \boldsymbol{r} \\
& +\int R_{0}^{2} v d \boldsymbol{r} \equiv E_{v}^{0}[\varphi],
\end{aligned}
$$


for the conserved (phase-independent) entropy $S_{0}\left[\varphi_{0}\right]=S^{\text {class. }}\left[p_{0}\right]$ in the system ground-state $\varphi_{0}(\boldsymbol{r})=R_{0}(\boldsymbol{r})$, for which $\phi(\boldsymbol{r})=\phi_{0}=0$, and hence $\nabla \phi_{0}=\boldsymbol{j}(\boldsymbol{r})=$ $\boldsymbol{j}_{0}(\boldsymbol{r})=\mathbf{0}$

$$
\begin{aligned}
& S^{\text {nclass. }}\left[\varphi_{0}\right] \equiv S\left[\phi_{0}\right]=I^{\text {nclass. }}\left[\varphi_{0}\right] \equiv I\left[\phi_{0}\right]=0 \text { or } \\
& S\left[\varphi_{0}\right]=S^{\text {class. }}\left[\varphi_{0}\right]=S\left[p_{0}\right] \quad \text { and } \quad I\left[\varphi_{0}\right]=I^{\text {class. }}\left[\varphi_{0}\right]=I\left[p_{0}\right] .
\end{aligned}
$$

This entropy/information-constrained, $S_{0}=S\left[\phi_{0}\right]=I_{0}=I\left[\phi_{0}\right]=0$, minimumenergy principle correctly predicts the molecular ground-state equilibrium:

$$
\begin{aligned}
\min _{\phi} E_{v}^{0}[\phi] & =\left\langle\phi_{0}|\hat{\mathrm{H}}| \phi_{0}\right\rangle=\left(\hbar^{2} / 2 m\right) \int\left(\nabla R_{0}\right)^{2} d \boldsymbol{r}+\int R_{0}^{2} v d \boldsymbol{r} \equiv E_{v}\left[\varphi_{0}\right] \\
& =\left(\frac{\hbar^{2}}{8 m}\right) \int \frac{\left(\nabla p_{0}\right)^{2}}{p_{0}} d \boldsymbol{r}+\int p_{0} v d \boldsymbol{r}=E_{v}\left[p_{0}\right]
\end{aligned}
$$

For this constrained value of system ground-state electronic energy, $E_{0}=E_{v}\left[p_{0}\right]$, this optimum solution also marks the maximum of the variational non-classical entropy $S\left[p_{0}, \phi\right]$ and the minimum of the associated density-constrained quantum Fisher information $I\left[p_{0}, \phi\right]$ :

$$
\max _{\phi} S\left[p_{0}, \phi\right]=S\left[p_{0}, \phi_{0}\right]=\min _{\phi} I\left[p_{0}, \phi\right]=I\left[p_{0}, \phi_{0}\right]=0 .
$$

Therefore, we have arrived at a remarkable parallelism with the ordinary thermodynamics: the ground-state stationary solution results from the equivalent electrondensity constrained (vertical) variational principles: of the system minimum electronic energy or the extremum of the quantum entropy/information.

Let us now examine the associated Euler equations determining the optimum orbital phase for the fixed ground-state probability distribution $p_{0}=R_{0}^{2}$ in this simplest, oneelectron case. One observes that the optimum solutions are derived from the extrema of the non-classical entropy/information functionals $S\left[p_{0}, \phi\right]$ and $I\left[p_{0}, \phi\right]$, for the fixed classical contributions $S\left[p_{0}\right]$ and $I\left[p_{0}\right]$, respectively. Since in quantum mechanics the phase of wave-functions is determined only up to an arbitrary constant, its sign is physically irrelevant. In what follows, we thus assume $\phi(\boldsymbol{r}) \equiv|\phi(\boldsymbol{r})|=\pi(\boldsymbol{r})^{1 / 2} \geq 0$. The vertical extrema of the two preceding equations,

$$
\max _{\phi} S\left[p_{0}, \phi\right] \text { or } \min _{\phi} I\left[p_{0}, \phi\right] \text {, }
$$

give rise to the following Euler equations for the optimum phase $\phi=\phi^{o p t}$. in the modulus-constrained trial state $\varphi^{0}(\boldsymbol{r})=\varphi\left[p_{0}, \phi ; \boldsymbol{r}\right]$ :

$$
\begin{aligned}
& \delta\left\langle\varphi^{0}\left|\hat{\mathbf{S}}_{\varphi}\right| \varphi^{0}\right\rangle /\left.\delta \varphi^{0}(\boldsymbol{r})^{*}\right|_{\varphi=\varphi^{o p t .}}=0 \quad \text { or } \quad \hat{\mathbf{S}}_{\varphi^{\text {opt. }}}(\boldsymbol{r}) \varphi_{0}(\boldsymbol{r})=-2 \phi^{o p t .}(\boldsymbol{r}) \varphi_{0}(\boldsymbol{r})=0 \\
& \delta\left\langle\varphi^{0}\left|\hat{\mathrm{I}}_{\varphi}\right| \varphi^{0}\right\rangle /\left.\delta \varphi^{0}(\boldsymbol{r})^{*}\right|_{\varphi=\varphi^{o p t .}}=0 \quad \text { or } \hat{\mathrm{I}}_{\varphi^{o p t .}}(\boldsymbol{r}) \varphi_{0}(\boldsymbol{r})=4\left[\nabla \phi^{o p t .}(\boldsymbol{r})\right]^{2} \varphi_{0}(\boldsymbol{r})=0 .
\end{aligned}
$$


Hence, both these (non-classical) extreme entropy/information rules properly predict the stationary, ground-state solution $\phi^{\text {opt }} \cdot(\boldsymbol{r})=\phi_{0}(\boldsymbol{r})=0$ as the vertical equilibrium state of this model system: $\varphi_{e q} .\left[p_{0}, \phi^{o p t} ; \boldsymbol{r}\right]=\varphi\left[p_{0}, \phi_{0} ; \boldsymbol{r}\right]=\varphi_{0}(\boldsymbol{r})$.

We thus conclude, that the non-classical, (phase/current)-related Extreme Information Principles (EPI) correctly identify the lowest eigenstate of this one-electron Hamiltonian as the vertical equilibrium state of the molecule, for the fixed groundstate probability distribution, in which all physical quantities become functionals of the system electron density alone, in accordance with the first Hohenberg-Kohn theorem of the modern DFT $[35,36]$. A generalization to $N>1$ case, using the determinantal wave-functions for the specified $\rho$ (or $p$ ) of Harriman [37], Zumbach and Maschke [38] (HZM), confirms this one-electron result [18,19] (see also the following two sections).

Let us similarly explore implications of the horizontal extrema of the resultant (quantum) entropy/information content, combining both the classical and non-classical contributions, subject only to the "geometric" constraint of the wave-function or probability normalization. The associated equilibrium states are now determined by the extrema of the corresponding auxiliary orbital functionals including the relevant information term and the constraint $\langle\varphi \mid \varphi\rangle=\int p(\boldsymbol{r}) d \boldsymbol{r}=1$ multiplied by the Lagrange multiplier $\mu$ enforcing this unit norm of the wave-function $\varphi$, i.e., the normalization of the electron probability density $p$ :

$$
\Omega_{S}[\varphi]=\langle\varphi|\hat{\mathrm{S}}| \varphi\rangle-\mu\langle\varphi \mid \varphi\rangle \quad \text { and } \quad \Omega_{I}[\varphi]=\langle\varphi|\hat{\mathrm{I}}| \varphi\rangle-\mu\langle\varphi \mid \varphi\rangle .
$$

The resulting Euler equations for the optimum orbital in this horizontal equilibrium state, expressed in terms of the system probability density $p$ and the equilibrium phase $\phi_{e q .}(\boldsymbol{r})=\phi_{e q .}[\boldsymbol{r} ; p]$

$$
\varphi_{e q .}\left[\boldsymbol{r} ; p, \phi_{e q .}[p]\right] \equiv \varphi_{e q .}[\boldsymbol{r} ; p]
$$

correspond to the vanishing functional derivative with respect to $\varphi^{*}(\boldsymbol{r})$ of the orbital functionals of Eq. (35):

$$
\begin{aligned}
& \delta \Omega_{S}[\varphi] /\left.\delta \varphi^{*}(\boldsymbol{r})\right|_{\phi=\phi_{e q .}}=\left\{\hat{\mathrm{S}}\left[\boldsymbol{r} ; p, \varphi_{e q .}[p]\right]-\mu\right\} \varphi_{e q .}(\boldsymbol{r})=0 \quad \text { or } \\
& \left\{-\ln p(\boldsymbol{r})-2 \phi_{e q .}[\boldsymbol{r} ; p]-\mu\right\} \varphi_{e q .}(\boldsymbol{r})=0, \quad \text { and } \\
& \delta \Omega_{I}[\varphi] /\left.\delta \varphi^{*}(\boldsymbol{r})\right|_{\phi=\phi_{e q .}}=\left\{\hat{\mathrm{I}}\left[\boldsymbol{r} ; p, \varphi_{e q .}[p]\right]-\mu\right\} \varphi_{e q .}(\boldsymbol{r})=0 \quad \text { or } \\
& \left\{[\nabla p(\boldsymbol{r}) / p(\boldsymbol{r})]^{2}+4\left[\nabla \phi_{e q .}(\boldsymbol{r})\right]^{2}-\mu\right\} \varphi_{e q .}(\boldsymbol{r})=0 .
\end{aligned}
$$

The first of these two horizontal principles predict a non-vanishing spatial phase related to the system probability distribution [16-19],

$$
\phi_{e q .}[\boldsymbol{r} ; p]=-(1 / 2) \ln p(\boldsymbol{r})+\text { const. } \equiv \phi_{e q .}(\boldsymbol{r})
$$

and hence:

$$
\varphi_{e q .}[\boldsymbol{r} ; p]=R(\boldsymbol{r}) \exp \left[\mathrm{i} \phi_{e q .}(\boldsymbol{r})\right]=R(\boldsymbol{r}) \exp [-(1 / 2) \ln p(\boldsymbol{r})] \equiv \varphi_{e q .}(\boldsymbol{r}) .
$$


We call such IT-equilibrium states, corresponding to the energy-unconstrained extrema of the resultant (quantum) entropy/information measures, the phase-equilibria [39] of the system under consideration. One also observes that the second principle [Eq. (37b)] does not have real solutions. This suggests a change of sign of the non-classical Fisher measure, by replacing $I^{\text {nclass. }} \cdot[\varphi]$ with $\tilde{I}^{\text {nclass } .}[\varphi]=-I^{\text {nclass. }}[\varphi]$ in the modified resultant gradient measure $\tilde{I}[\varphi]=I^{\text {class }} \cdot[\varphi]+\tilde{I}^{\text {nclass }} \cdot[\varphi]$, after which one again recovers the equilibrium phase of Eq. (39) as the optimum "thermodynamic" solution.

\section{Molecular equilibria in $N$-electron systems}

Next, let us similarly examine the relevant equilibrium states in many-electron systems. In modern DFT $[35,36]$ one often refers to the vertical states and principles $[10-13,18,19,37,40-43]$ corresponding to the fixed density or probability distribution of electrons, e.g., in Levy's [40] construction of the universal density functional for the sum of the electron kinetic and repulsion energies. They give rise to the corresponding vertical IT equilibria, which are solely determined by the extrema of the non-classical entropy/information functionals. Accordingly, the variational principles of the resultant information measure (density unrestricted) similarly determine the horizontal phase equilibria in molecules.

A related problem of constructing the antisymmetric wave function of $N$ fermions yielding the prescribed density $\rho(\boldsymbol{r})$, vital for solving the familiar $N$-representability problem of DFT, has been tackled by Harriman [37] using crucial insights due to Macke [41] and Gilbert [42]. Its three-dimensional generalization by Zumbach and Maschke [38] introduces the complete set of the density-conserving Slater determinants. They are build using the (plane-wave)-type equidensity orbitals

$$
\left\{\varphi_{\boldsymbol{k}}(\boldsymbol{r})=R(\boldsymbol{r}) \exp \left[\mathrm{i} \Phi_{\boldsymbol{k}}(\boldsymbol{r})\right]\right\}
$$

which offer a convenient framework for an extension of the above one-particle IT analysis to general $N$-electron systems [18,19].

In constructing the orthogonal Slater determinants that generate the specified electron density these orbitals adopt equal, density-dependent modulus $R(\boldsymbol{r})=p(\boldsymbol{r})^{1 / 2}$ and the spatial phase

$$
\Phi_{k}(\boldsymbol{r})=\boldsymbol{k} \cdot \boldsymbol{f}(\boldsymbol{r})+\phi(\boldsymbol{r}) \equiv F_{k}(\boldsymbol{r})+\phi(\boldsymbol{r}),
$$

with the density-dependent vector function $\boldsymbol{f}(\boldsymbol{r})=\boldsymbol{f}[\boldsymbol{r} ; p]$, common to all equidensity orbitals, linked to the Jacobian of the $\boldsymbol{r} \rightarrow \boldsymbol{f}(\boldsymbol{r})$ transformation.

The optimum (orthonormal) HZM orbitals [37,38] for the specified ground-state probability distribution $p_{0}(\boldsymbol{r})=\left[R_{0}(\boldsymbol{r})\right]^{2}$,

$$
\begin{aligned}
\varphi_{\boldsymbol{k}}(\boldsymbol{r}) & =\left[p_{0}(\boldsymbol{r})\right]^{1 / 2} \exp \left\{\mathrm{i}\left[\boldsymbol{k} \cdot \boldsymbol{f}\left[\boldsymbol{r} ; p_{0}\right]+\phi(\boldsymbol{r})\right]\right\} \\
& \equiv R_{0}(\boldsymbol{r}) \exp \left(\mathrm{i} \Phi_{\boldsymbol{k}}\left[\boldsymbol{r} ; p_{0}\right]\right) \equiv \varphi_{\boldsymbol{k}}\left[\boldsymbol{r} ; p_{0}\right],
\end{aligned}
$$


are shaped by the "orthogonality" phase $F_{\boldsymbol{k}}\left[\boldsymbol{r} ; p_{0}\right]=\boldsymbol{k} \cdot \boldsymbol{f}\left[\boldsymbol{r} ; p_{0}\right]$, with the wave-vector (reduced momentum) $\boldsymbol{k}$ and the the density-dependent spatial vector field $\boldsymbol{f}_{0}(\boldsymbol{r})=$ $\boldsymbol{f}\left[\boldsymbol{r} ; p_{0}\right]$ resulting from the ordinary variational principle for the system minimum electronic energy, e.g., in the familiar Self-Consistent Field (SCF) theories $[36,44,45]$. The optimum form of the remaining part $\phi(\boldsymbol{r})$ of $\Phi_{\boldsymbol{k}}(\boldsymbol{r})$, called "thermodynamic" phase [19], results from the quantum Extreme Physical Information (EPI) rule, for the given ground-state density $\rho=\rho_{0}$ or the associated probability distribution $p=p_{0}$. The latter are determined at the energy optimization stage, which generally gives $\phi^{o p t} \cdot(\boldsymbol{r})=$ $\phi_{e q .}\left[\boldsymbol{r} ; p_{0}\right]$ and hence $\boldsymbol{j}\left[\boldsymbol{r} ; \varphi_{e q} .\left[p_{0}\right]\right] \neq 0$. Therefore, the horizontal equilibrium state of $N$ electrons again implies a non-vanishing phase-gradient and hence also a presence of a finite probability current.

The spatial phase function $\Phi_{\boldsymbol{k}}(\boldsymbol{r})$ of equidensity orbitals thus involves the (orbitalspecific) orthogonality (geometric) contribution $\boldsymbol{k} \cdot \boldsymbol{f}(\boldsymbol{r}) \equiv F_{\boldsymbol{k}}(\boldsymbol{r})$, which enforces the independence of these one-particle states, and a "thermodynamic" term $\phi(r)$ common to all orbitals. The Slater determinants build from the specific selection of $N$ different equidensity orbitals,

$$
\Psi_{\boldsymbol{k}_{1}, \boldsymbol{k}_{2}, \ldots, \boldsymbol{k}_{N}}(N)=(1 / \sqrt{N !}) \operatorname{det}\left(\varphi_{\boldsymbol{k}_{1}}, \varphi_{\boldsymbol{k}_{2}}, \ldots, \varphi_{\boldsymbol{k}_{N}}\right) \equiv \Psi_{\mathbf{k}}(N), \boldsymbol{k}_{i} \neq \boldsymbol{k}_{j} \text { for } i \neq j
$$

then by construction reproduce the prescribed electron density $\rho(\boldsymbol{r})$,

$$
\rho(\boldsymbol{r})=N p(\boldsymbol{r})=\sum_{i=1}^{N}\left|\varphi_{\boldsymbol{k}_{i}}(\boldsymbol{r})\right|^{2}
$$

They constitute the complete orthonormal system of $N$-particle functions capable of representing any molecular state of $N$ electrons for the specified electron distribution $p(\boldsymbol{r})$, in the HZM Configuration-Interaction (CI) expansion:

$$
\Psi_{0}(N)=\int C_{0}(\mathbf{k}) \Psi_{\mathbf{k}}(N) d \mathbf{k}
$$

Let us explore the relation between the (horizontal) equilibrium phase of orbitals and the molecular probability distribution in general many-electron systems. In the single-determinant approximation,

$$
\begin{aligned}
\Psi_{\mathbf{k}} & =\Psi_{\boldsymbol{k}_{1}, \boldsymbol{k}_{2}, \ldots, \boldsymbol{k}_{N}}=\Psi[N ; p]=(N !)^{-1 / 2} \operatorname{det}\left(\left\{\varphi_{l}[p]\right\}\right), \\
\varphi_{l}[p] & =[p(\boldsymbol{r})]^{1 / 2} \exp \left[\mathrm{i} \Phi_{l}[\boldsymbol{r} ; p]\right], \\
\Phi_{l}[\boldsymbol{r} ; p] & =\Phi_{l}[\boldsymbol{r} ; \boldsymbol{k}[p], p]=\boldsymbol{k}_{l}[p] \cdot f[\boldsymbol{r} ; p]+\phi_{e q}[\boldsymbol{r} ; N, p] \equiv \Phi_{l}(\boldsymbol{r}) \geq 0,
\end{aligned}
$$

the average quantum entropy or the associated overall gradient measure of the information content are given by the sums of the corresponding orbital contributions, the orbital expectation values of the one-electron entropy/information operators: 


$$
\begin{aligned}
& S[\Psi[p]]=\sum_{l}\left\langle\varphi_{l}[p]|\hat{\mathrm{S}}| \varphi_{l}[p]\right\rangle=\sum_{l} S\left[\varphi_{l}[p]\right] \quad \text { or } \\
& I[\Psi[p]]=\sum_{l}\left\langle\varphi_{l}[p]|\hat{\mathrm{I}}| \varphi_{l}[p]\right\rangle=\sum_{l} I\left[\varphi_{l}[p]\right] .
\end{aligned}
$$

For equidensity orbitals the classical, modulus-related contributions are identical, so that the overall probability-entropy in $\Psi(N)$ reads:

$$
S^{\text {class. }}[\Psi[p]]=\sum_{l}\left\langle\varphi_{l}[p]\left|\hat{S}_{p}\right| \varphi_{l}[p]\right\rangle=N S[p] .
$$

The phase-entropy now inculdes the following average orthogonality and thermodynamic contributions of $N$ electrons:

$$
\begin{aligned}
S^{\text {nclass. }[\Psi[N, p]]} & =\sum_{l}\left\langle\varphi_{l}[p]\left|\hat{\mathrm{S}}_{\Phi}\right| \varphi_{l}[p]\right\rangle=-2 \int p(\boldsymbol{r})\left\{\sum_{l} \Phi_{l}[\boldsymbol{r} ; N, p]\right\} d \boldsymbol{r} \\
& =-2 \int p(\boldsymbol{r})\left(\sum_{l} \boldsymbol{k}_{l} \cdot \boldsymbol{f}[\boldsymbol{r} ; p]\right) d \boldsymbol{r}-2 \sum_{l} \int p(\boldsymbol{r}) \phi_{e q .}[\boldsymbol{r} ; N, p] d \boldsymbol{r} \\
& \equiv-2 N \int p(\boldsymbol{r})\left\{\boldsymbol{K}(\mathbf{k}[p]) \cdot \boldsymbol{f}[\boldsymbol{r} ; p]+\phi_{e q .}[\boldsymbol{r} ; N, p]\right\} d \boldsymbol{r}
\end{aligned}
$$

where the average "wave-number" vector of $\Psi_{\mathbf{k}}$

$$
\boldsymbol{K}(\mathbf{k}[p])=N^{-1} \sum_{l} \boldsymbol{k}_{l}[p] .
$$

The horizontal equilibria mark the extrema of the auxiliary entropy/information functionals with respect to thermodynamic phase:

$$
\delta\{S[\Psi]-\mu\langle\Psi \mid \Psi\rangle\}=0 \quad \text { or } \quad \delta\{\tilde{I}[\Psi]-\mu\langle\Psi \mid \Psi\rangle\}=0, \quad\langle\Psi \mid \Psi\rangle=\prod_{l}\left\langle\varphi_{l} \mid \varphi_{l}\right\rangle=1
$$

where $\tilde{I}[\Psi] \equiv\langle\Psi(N)|\hat{\tilde{I}}(N)| \Psi(N)\rangle=I^{\text {class. }}[\Psi]+\tilde{I}^{\text {nclass. }}[\Psi]=I^{\text {class. }}[\Psi \Psi]$ $-I^{\text {nclass }} \cdot[\Psi] \equiv \sum_{l}\left\langle\varphi_{l}[p]|\hat{\tilde{I}}| \varphi_{l}[p]\right\rangle$.

By performing independent variations $\left\{\delta \varphi_{l}^{*}\right\}$ of the complex-conjugate orbitals these principles give:

$$
\sum_{l}\left\langle\delta \varphi_{l}|\hat{\mathrm{S}}-\mu| \varphi_{l}\right\rangle=0 \quad \text { or } \quad \sum_{l}\left\langle\delta \varphi_{l}|\hat{\tilde{\mathrm{I}}}-\mu| \varphi_{l}\right\rangle=0 .
$$

For arbitrary variations these relations imply the following Euler equations,

$$
[\hat{\mathrm{S}}-\mu] \varphi_{l}^{e q .}=0 \quad \text { or } \quad[\hat{\tilde{\mathrm{I}}}-\mu] \varphi_{l}^{e q .}=0, \quad l=1,2, \ldots, N
$$

to be satisfied by the optimum, equilibrium equidensity orbitals. 
For example, the horizontal principle of the stationary (resultant) quantum entropy now reads:

$$
\left\{-\ln p(\boldsymbol{r})-2 \boldsymbol{K}(\mathbf{k}[p]) \cdot \boldsymbol{f}[\boldsymbol{r} ; p]-2 \phi_{l}^{e q \cdot}[\boldsymbol{r} ; p]-\mu\right\} \varphi_{l}^{e q \cdot}(\boldsymbol{r})=0 .
$$

It generates the optimum (probability-related) thermodynamic phase,

$$
\begin{aligned}
\phi_{l}^{e q \cdot}[\boldsymbol{r} ; p] & =-(1 / 2) \ln p(\boldsymbol{r})-\boldsymbol{K}(\mathbf{k}[p]) \cdot \boldsymbol{f}[\boldsymbol{r} ; p]+\text { const. } \\
& \equiv-(1 / 2) \ln p(\boldsymbol{r})-\boldsymbol{K}(\mathbf{k}[p]) \cdot \boldsymbol{f}[\boldsymbol{r} ; p]=\phi_{e q .}[\boldsymbol{r} ; N, p],
\end{aligned}
$$

where we have again absorbed the constant phase contribution, irrelevant in quantum mechanics, by an appropriate choice of the zero value of orbital phases. It additionally includes the second, "orthogonality" contribution, which was missing in the oneelectron case [Eq. (38)]. Therefore, the resultant phase of the equilibrium equidensity orbital $\varphi_{l}^{e q .}$ reads:

$$
\begin{aligned}
\Phi_{l}[\boldsymbol{r} ; N, p] & =\boldsymbol{k}_{l}[p] \cdot \boldsymbol{f}[\boldsymbol{r} ; p]+\phi_{e q .}[\boldsymbol{r} ; N, p] \\
& =\left\{\boldsymbol{k}_{l}[p]-\boldsymbol{K}(\mathbf{k}[p])\right\} \cdot \boldsymbol{f}[\boldsymbol{r} ; p]-(1 / 2) \ln p(\boldsymbol{r}) \\
& \equiv \delta \boldsymbol{k}_{l}[p] \cdot \boldsymbol{f}[\boldsymbol{r} ; p]-(1 / 2) \ln p(\boldsymbol{r}) \equiv F_{l}[\boldsymbol{r} ; p]-(1 / 2) \ln p(\boldsymbol{r}) .
\end{aligned}
$$

The same prediction follows from the modified average Fisher information $\tilde{I}[\Psi]$. It also generates equal classical contributions from each equidensity orbital,

$$
I^{\text {class. }}[\Psi[p]]=\sum_{l}\left\langle\varphi_{l}[p]\left|\hat{\mathrm{I}}_{p}\right| \varphi_{l}[p]\right\rangle=N I[p],
$$

and the following non-classical contributions due to orbital orthogonality and thermodynamic phase:

$$
\begin{aligned}
\tilde{I}^{\text {nclass. }[\Psi[N, p]]} & =\sum_{l}\left\langle\varphi_{l}[p]\left|\hat{\tilde{I}}_{\Phi}\right| \varphi_{l}[p]\right\rangle=-4 \int p(\boldsymbol{r})\left\{\sum_{l} \nabla \Phi_{l}[\boldsymbol{r} ; N, p]\right\}^{2} d \boldsymbol{r} \\
& =-4 \int p(\boldsymbol{r})\left\{\nabla \sum_{l}\left(\boldsymbol{k}_{l} \cdot \boldsymbol{f}[\boldsymbol{r} ; p]+\phi_{\text {eq. }}[\boldsymbol{r} ; N, p]\right)\right\}^{2} d \boldsymbol{r} \\
& \equiv-4 N^{2} \int p(\boldsymbol{r})\left\{\nabla\left(\boldsymbol{K}\left(\mathbf{k}[p] \cdot \boldsymbol{f}[\boldsymbol{r} ; p]+\phi_{e q .}[\boldsymbol{r} ; N, p]\right)\right\}^{2} d \boldsymbol{r},(58)\right.
\end{aligned}
$$

where $\nabla(\boldsymbol{K} \cdot \boldsymbol{f})=\sum_{\alpha} K_{\alpha} \nabla f_{\alpha}$.

The horizontal principle of the stationary (modified) resultant Fisher information $\tilde{I}[\Psi]$ now gives the following Euler equation involving relevant gradient squares:

$$
\left\{[\nabla \ln p(\boldsymbol{r})]^{2}-4\left(\nabla\left[\boldsymbol{K}(\mathbf{k}[p]) \cdot \boldsymbol{f}[\boldsymbol{r} ; p]+\phi_{l}^{e q \cdot}[\boldsymbol{r} ; p]\right]\right)^{2}-\mu\right\} \varphi_{l}^{e q \cdot}(\boldsymbol{r})=0 .
$$


It implies the associated relation between the gradients themselves:

$$
\begin{aligned}
& -\nabla \ln p(\boldsymbol{r})=2 \nabla\left\{\boldsymbol{K}(\mathbf{k}[p]) \cdot \boldsymbol{f}[\boldsymbol{r} ; p]+\phi_{l}^{e q \cdot}[\boldsymbol{r} ; p]\right\} \quad \text { or } \\
& -\ln p(\boldsymbol{r})=2\left\{\boldsymbol{K}(\mathbf{k}[p]) \cdot \boldsymbol{f}[\boldsymbol{r} ; p]+\phi_{l}^{e q \cdot}[\boldsymbol{r} ; p],\right.
\end{aligned}
$$

which again determines the equilibrium thermodynamic phase of Eq. (55).

Therefore, the (horizontal) phase-equilibrium marks the resultant orbital phases determined by the electron probability distribution alone. For $p=p_{0}$ this is a manifestation of the Hohenberg-Kohn theorem [35]: the electron density uniquely determines the equilibrium equidensity orbitals:

$$
\mathbf{k}_{e q .}=\mathbf{k}\left[p_{0}\right], \boldsymbol{f}_{e q .}=\boldsymbol{f}\left[p_{0}\right], \phi_{e q .}=\phi_{e q .}\left[p_{0}\right]
$$

These spatial phases imply equal thermodynamic contribution,

$$
\boldsymbol{j}_{l}\left[\boldsymbol{r} ; \phi_{e q}\right]=-\frac{\hbar}{2 m} \nabla p(\boldsymbol{r})
$$

to the overall current density in the spin-orbital $\varphi_{l}^{e q .}(\boldsymbol{r})$,

$$
\boldsymbol{j}_{l}(\boldsymbol{r})_{e q .}=\frac{\hbar p(\boldsymbol{r})}{m} \nabla\left(\delta \boldsymbol{k}_{l}[p] \cdot \boldsymbol{f}[\boldsymbol{r} ; p]\right)+\boldsymbol{j}\left[\boldsymbol{r} ; \phi_{e q}\right]=\boldsymbol{j}_{l}\left[\boldsymbol{r} ; \boldsymbol{k}_{l}\right]+\boldsymbol{j}_{l}\left[\boldsymbol{r} ; \phi_{e q}\right]
$$

The resultant thermodynamic current of $N$ electrons is thus shaped by the density gradient:

$$
\boldsymbol{j}\left[\boldsymbol{r} ; \phi_{e q}\right]=\sum_{l} \boldsymbol{j}_{l}\left[\boldsymbol{r} ; \phi_{e q}\right]=N \boldsymbol{j}_{l}\left[\boldsymbol{r} ; \phi_{e q}\right]=-\frac{\hbar}{2 m} \nabla \rho(\boldsymbol{r}),
$$

while the "orthogonality" phases give rise to the vanishing average:

$$
\boldsymbol{j}[\boldsymbol{r} ; \mathbf{k}, p]=\sum_{l} \boldsymbol{j}_{l}\left[\boldsymbol{r} ; \boldsymbol{k}_{l}\right]=\frac{\hbar p(\boldsymbol{r})}{m} \nabla\left\{\left(\sum_{l} \delta \boldsymbol{k}_{l}[p]\right) \cdot \boldsymbol{f}[\boldsymbol{r} ; p]\right\}=0
$$

since $\sum_{l} \delta \boldsymbol{k}_{l}=\boldsymbol{0}$. Therefore, in the (horizontal) phase-equilibrium state of $N$ eletrons, the direction of the average resultant current is determined by the negative gradient of the molecular electron density, in full agreement with the Hohenberg-Kohn theorem [35].

To summarize: the phase side of the molecular electronic structure reflects its IT "entropic" aspect. The complex orbitals in the system (horizontal) phase equilibrium have been shown to exhibit a non-vanishing particle current, due to the state thermodynamic phase $\phi_{e q} .[r ; N, p]$. This state is thus qualitatively different from the lowest eigenstate (assumed non-degenerate) of the $N$-electron Hamiltonian, 


$$
\hat{\mathrm{H}}(N)=\hat{\mathrm{V}}_{n e}(N)+\left[\hat{\mathrm{T}}(N)+\hat{\mathrm{V}}_{e e}(N)\right] \equiv \sum_{i=1}^{N} v(i)+\hat{\mathrm{F}}(N),
$$

where $\hat{\mathrm{F}}(N)$ combines the electron kinetic $(\hat{\mathrm{T}})$ and repulsion $\left(\hat{\mathrm{V}}_{e e}\right)$ energy operators, in which the probability current identically vanishes.

Such an "entropic" interpretation has been also attributed [11-13] to the densityconstrained principles of modern DFT, in the vertical (entropic) searches performed for the specified electron density $\rho$, e.g., in Levy's [40] constrained-search construction of the universal part of the energy density functional,

$$
F[\rho]=T[\rho]+V_{e e}[\rho]=\inf _{\Psi \rightarrow \rho}\langle\Psi|\hat{\mathrm{F}}| \Psi\rangle \equiv\langle\Psi[\rho]|\hat{\mathrm{F}}| \Psi[\rho]\rangle .
$$

In this variational procedure one searches over the wave functions $\Psi(N)$ of $N$ electrons, which yield the given electron density $\rho$, symbolically denoted by $\Psi \rightarrow \rho$, and calculates the $v$-independent part $F[\rho]$ of the density functional for the system overall electronic energy,

$$
E_{v}[\rho]=\int v(\boldsymbol{r}) \rho(\boldsymbol{r}) d \boldsymbol{r}+F[\rho]
$$

as the lowest value (infimum) of the expectation value of $\hat{\mathrm{F}}(N)$. When this search is performed for the fixed ground-state density $\rho=\rho_{0}=N p_{0}$ it also implies the fixed DFT value $E_{v}\left[\rho_{0}\right]$ of the system electronic energy, by the first Hohenberg-Kohn (HK) theorem [35]. Notice, however, that only at the exact ground-state $\Psi\left[\rho_{0}\right]$ the expectation value of the system energy recovers the DFT energy level:

$$
E_{v}\left[\rho_{0}\right]=\left\langle\Psi\left[\rho_{0}\right]|\hat{\mathrm{H}}| \Psi\left[\rho_{0}\right]\right\rangle \leq\left\langle\Psi \rightarrow \rho_{0}|\hat{\mathrm{H}}| \Psi \rightarrow \rho_{0}\right\rangle .
$$

This feature is reminiscent of the classical criterion for determining the equilibrium state formulated in the entropy representation of the ordinary thermodynamics [33], viz., the maximum-entropy principle for constant internal energy. Indeed, in accordance with the second $\mathrm{HK}$ theorem [35] the familiar variational principle for determining the ground-state wave-function at the minimum of the system energy can be interpreted as the DFT optimization over all admissible densities. It involves the "internal" (entropic) search over functions of $N$ fermions that yield the current trial density of the "external" (energetic) search:

$$
\min _{\Psi}\langle\Psi|\hat{\mathrm{H}}| \Psi\rangle=\min _{\rho} E_{v}[\rho]=\min _{\rho}\left\{\int v(\boldsymbol{r}) \rho(\boldsymbol{r}) d \boldsymbol{r}+\inf _{\Psi \rightarrow \rho}\langle\Psi|\hat{\mathrm{F}}| \Psi\rangle\right\}
$$

Consider now the equilibrium principles for the vertical extrema of the system entropy or information, corresponding to the fixed ground-state electron density $\rho=\rho_{0}$ or its probability factor $p=\rho_{0} / N=p_{0}$ determined in the external search of the preceding equation. In DFT the internal, entropic principle involves the search for the optimum 
wave function $\Psi(N)$ corresponding to the fixed external potential $v$ due to the system nuclei:

$$
\begin{aligned}
E\left[\Psi\left[\rho_{0}\right]\right] & =E_{v}\left[\rho_{0}\right]=\int v(\boldsymbol{r}) \rho_{0}(\boldsymbol{r}) d \boldsymbol{r}+\inf _{\Psi \rightarrow \rho_{0}}\langle\Psi|\hat{\mathrm{F}}| \Psi\rangle \\
& =\int v(\boldsymbol{r}) \rho_{0}(\boldsymbol{r}) d \boldsymbol{r}+F\left[\rho_{0}\right] \\
& =\int v(\boldsymbol{r}) \rho_{0}(\boldsymbol{r}) d \boldsymbol{r}+V_{e e}\left[\rho_{0}\right]+\inf _{\Psi \rightarrow \rho_{0}}\langle\Psi|\hat{\mathrm{T}}| \Psi\rangle
\end{aligned}
$$

One observes a presence of Levy's universal functional $F[\rho]$ as the crucial (entropic) part of this information principle. Notice also that in DFT the external potential and electron-repulsion energies are fixed by the frozen ground-state density, so that the optimum state also marks the infimum of the Fisher measure of the information content related to the system average kinetic energy.

Consider the resultant Fisher information in the electronic state approximated by a single Harriman determinant of Eq. (43), $\Psi(N) \approx \Psi_{\mathbf{k}}(N)$,

$$
\begin{aligned}
I\left[\Psi_{\mathbf{k}}\right] & =4 \sum_{i=1}^{N} \int\left|\nabla \phi_{\boldsymbol{k}_{i}}(\boldsymbol{r})\right|^{2} d \boldsymbol{r}=\frac{8 m}{\hbar^{2}} T\left[\Psi_{\mathbf{k}}\right] \equiv \int p(\boldsymbol{r}) I(\boldsymbol{r}) d \boldsymbol{r} \\
& =\int p(\boldsymbol{r})\left[I^{\text {class. }}(\boldsymbol{r})+I^{n \text { class. }}(\boldsymbol{r})\right] d \boldsymbol{r}=I[p]+I[p, \mathbf{k}, \phi],
\end{aligned}
$$

proportional to the system average kinetic Energy

$$
\begin{aligned}
T\left[\Psi_{\mathbf{k}}\right] & \equiv\left\langle\Psi_{\mathbf{k}}|\hat{\mathrm{T}}| \Psi_{\mathbf{k}}\right\rangle=\frac{\hbar^{2}}{2 m} \sum_{i=1}^{N} \int\left|\nabla \phi_{\boldsymbol{k}_{i}}(\boldsymbol{r})\right|^{2} d \boldsymbol{r} \\
& \equiv T^{\text {class. }}\left[\Psi_{\mathbf{k}}\right]+T^{\text {nclass. }}\left[\Psi_{\mathbf{k}}\right] \equiv T[p]+T[p, \mathbf{k}, \phi]
\end{aligned}
$$

The latter consists of the "classical”, von Weizscker's density functional $T[p]$, depending solely upon the particle distribution,

$$
T^{\text {class. }}\left[\Psi_{\mathbf{k}}\right]=\frac{\hbar^{2} N}{8 m} \int \frac{|\nabla p(\boldsymbol{r})|^{2}}{p(\boldsymbol{r})} d \boldsymbol{r}=T[p],
$$

and the "non-classical", (phase/current)-dependent contribution,

$$
T^{n \text { class. }}\left[\Psi_{\mathbf{k}}\right]=\frac{\hbar^{2}}{2 m} \int p(\boldsymbol{r}) \sum_{l=1}^{N}\left(\nabla\left[\boldsymbol{k}_{l} \cdot \boldsymbol{f}(\boldsymbol{r})+\phi(\boldsymbol{r})\right]\right)^{2} d \boldsymbol{r}=T[p, \mathbf{k}, \phi],
$$

related to the corresponding quantum-information terms of Eq. (72).

In the vertical (ground-state) search, for $p=p_{0}=\rho_{0} / N$, it is the phase component of the quantum state which is being optimized. The condition of the extremum (minimum) Fisher information $I\left[\Psi_{\mathbf{k}}\left[p_{0}\right]\right]$, 


$$
\begin{aligned}
& \delta I\left[\Psi_{\mathbf{k}}\left[p_{0}\right]\right] / \delta \phi(\boldsymbol{r})=\partial I\left[p_{0}, \phi\right] / \partial \phi(\boldsymbol{r})=0 \\
& \text { or } \sum_{l=1}^{N}\left\{\boldsymbol{k}_{l} \cdot \boldsymbol{f}\left[\boldsymbol{r} ; p_{0}\right]+\phi^{e q \cdot}\left[\boldsymbol{r} ; p_{0}\right]\right\}=0,
\end{aligned}
$$

determines the equilibrium thermodynamic phase $\phi^{e q} \cdot\left[p_{0} ; \boldsymbol{r}\right]$ that minimizes $I\left[p_{0}, \phi\right]$,

$$
\phi^{e q \cdot}\left[\boldsymbol{r} ; p_{0}\right]=-\left(\frac{1}{N} \sum_{l=1}^{N} \boldsymbol{k}_{l}\right) \cdot \boldsymbol{f}\left[\boldsymbol{r} ; p_{0}\right] \equiv-\boldsymbol{K}(\mathbf{k}) \cdot \boldsymbol{f}\left[\boldsymbol{r} ; p_{0}\right]
$$

It is seen to contain only the second, "orthogonality" contribution of Eq. (55). Therefore, the vertical equilibrium phase is determined by the average "wave-number" vector $\boldsymbol{K}$ in $\Psi_{\mathbf{k}}=\Psi_{\boldsymbol{k}_{1}, \boldsymbol{k}_{2}, \ldots, \boldsymbol{k}_{N}}$, for which the density of $I^{\text {nclass. }}$ exhibits the least structure, i.e., the maximum indeterminacy. Hence, the vertical-equilibrium equidensity orbitals,

$$
\begin{aligned}
\varphi_{\boldsymbol{k}_{l}}\left[\boldsymbol{r} ; p_{0}\right] & =\left[p_{0}(\boldsymbol{r})\right]^{1 / 2} \exp \left(\mathrm{i}\left\{\left(\boldsymbol{k}_{l}-\boldsymbol{K}\right) \cdot \boldsymbol{f}\left[\boldsymbol{r} ; p_{0}\right]\right\}\right) \\
& \equiv\left[p_{0}(\boldsymbol{r})\right]^{1 / 2} \exp \left\{\mathrm{i} \delta \boldsymbol{k}_{l} \cdot \boldsymbol{f}\left[\boldsymbol{r} ; p_{0}\right]\right\},
\end{aligned}
$$

lead to the identically vanishing non-classical kinetic energy

$$
T^{\text {nclass. }}\left[p_{0}, \phi^{e q \cdot}\left[p_{0}\right]\right]=\frac{\hbar^{2}}{2 m} \int p_{0}(\boldsymbol{r})\left(\nabla\left\{\left[\sum_{l} \delta \boldsymbol{k}_{l}\right] \cdot \boldsymbol{f}\left[\boldsymbol{r} ; p_{0}\right]\right\}\right)^{2} d \boldsymbol{r}=0
$$

due to a vanishing overall "orthogonality" current [Eq. (65)]:

$$
\boldsymbol{j}\left[\boldsymbol{r} ; \mathbf{k}, p_{0}\right]=\frac{\hbar}{m} p_{0}(\boldsymbol{r}) \nabla\left(\left[\sum_{l=1}^{N} \delta \boldsymbol{k}_{l}\right] \cdot \boldsymbol{f}\left[\boldsymbol{r} ; p_{0}\right]\right)=\mathbf{0} .
$$

To conclude this section, let us summarize these energy/information principles for the one-determinantal HZM approximation of the system ground-state wave function $\Psi_{0}(N)=\Psi[N, v]$ :

$$
\Psi_{0}(N) \cong \Psi_{\mathbf{k}_{0}}\left[N, \varphi_{0}\right] \equiv \Psi_{\mathbf{k}_{0}}(N) \quad \Rightarrow \quad \rho_{0}=N p_{0}
$$

e.g., in the Kohn-Sham (KS) [36] or Hartree-Fock (HF) [44,45] SCF theories. For the stationary molecular states, corresponding to the vanishing thermodynamic phase $\phi=0 \equiv \phi_{0}$, the optimum (real) reduced-momentum vectors $\mathbf{k}\left[p_{0}\right] \equiv \mathbf{k}_{0}\left[p_{0}\right]=$ $\left\{\boldsymbol{k}_{l}^{0}\left[p_{0}\right]\right\}$ result from the (vertical) minimum energy principle determining the best (orthonormal) equidensity orbitals $\varphi_{\boldsymbol{k}}\left[p_{0} ; \boldsymbol{r}\right]$ that minimize the average value of the system electronic energy:

$$
\begin{aligned}
& \min _{\Psi_{\mathbf{k}} \rightarrow \rho_{0}}\left\langle\Psi_{\mathbf{k}}(N)|\hat{\mathrm{H}}(N)| \Psi_{\mathbf{k}}(N)\right\rangle \\
& \quad=\left\langle\Psi_{\mathbf{k}_{0}\left[p_{0}\right]}(N)|\hat{\mathrm{H}}(N)| \Psi_{\mathbf{k}_{0}\left[p_{0}\right]}(N)\right\rangle=E_{\mathbf{k}_{0}\left[p_{0}\right]}(N) \Rightarrow \Psi_{\mathbf{k}_{0}\left[p_{0}\right]}(N) .
\end{aligned}
$$


The optimum (stationary-state) determinant $\Psi_{\mathbf{k}_{0}\left[p_{0}\right]}(N)$ then generates the best variational estimate $E_{\mathbf{k}_{0}\left[p_{0}\right]}(N)$ of the ground-state energy $E_{0}=E_{v}\left[\rho_{0}\right]$ in the HZM representation. This energy variational procedure implies the typical Euler-Lagrange problem of the auxiliary energy functional absorbing the orbital orthonormality constraints enforced by the relevant Lagrange multipliers.

The associated (vertical) equilibrium state $\Psi_{\mathbf{k}_{0}\left[p_{0}\right]}\left[N, \varphi^{e q \cdot}\left[p_{0}\right]\right]$ results from the extra (Fisher) EPI:

$$
\begin{aligned}
\inf _{\Psi \rightarrow \rho_{0}} I[\Psi]= & I^{\text {class. }}\left[p_{0}\right]+\inf _{\Psi \rightarrow \rho_{0}} I^{\text {nclass. }}\left[p_{0}, \mathbf{k}_{0}\left[p_{0}\right], \phi\right] \\
& \Rightarrow \Psi_{\mathbf{k}_{0}\left[p_{0}\right]}\left[N, \varphi^{e q \cdot} \cdot\left[p_{0}\right]\right] .
\end{aligned}
$$

In the HZM construction the lowest equilibrium state is thus determined by the best SCF values of the "wave-number" vectors $\mathbf{k}_{0}\left[p_{0}\right]=\left\{\boldsymbol{k}_{l}^{0}\left[p_{0}\right]\right\}$ of the energy variational principle of Eq. (82) and the optimum thermodynamic phase due to the orbital orthogonality [Eq. (77)]

$$
\phi(\boldsymbol{r})=\phi^{e q} \cdot\left[\boldsymbol{r} ; p_{0}\right]=-\left(\frac{1}{N} \sum_{l=1}^{N} \boldsymbol{k}_{l}^{0}\left[p_{0}\right]\right) \cdot \boldsymbol{f}_{0}(\boldsymbol{r}) \equiv-\boldsymbol{K}\left(\mathbf{k}_{0}\left[p_{0}\right]\right) \cdot \boldsymbol{f}_{0}\left[\boldsymbol{r} ; p_{0}\right]
$$

Together they uniquely specify the $N$ lowest, singly-occupied (complex), othonormal spin-orbitals of the vertical-equilibrium Slater determinant $\Psi_{\mathbf{k}_{0}\left[p_{0}\right]}\left[N, \varphi^{e q \cdot}\left[p_{0}\right]\right]$. The minimum Fisher information principle thus involves a search for the optimum equidensity orbitals of $N$ electrons in the ground-state electron distribution $\rho_{0}=N p_{0}$, determined by the energy-optimum "wave-number" vectors $\mathbf{k}_{0}\left[p_{0}\right]$ and the equilibrium phase $\phi^{e q .}\left[p_{0}\right]$ [see Eq.(78)].

The classical and non-classical parts of the densities-per-electron of the quantum measures of the resultant Fisher and Shannon information descriptors are mutually related, with the former being determined by the squared gradient of the latter [Eq. (28)]. One further observes that for the fixed ground-state distribution of electrons, in the "internal" (vertical) search, only the non-classical components depend upon the "wave-number" vectors $\mathbf{k}_{0}\left[p_{0}\right] \equiv \mathbf{k}_{0}$ and the thermodynamic phase function $\phi(r)$, which together determine the resultant phase in Harriman's construction, to be optimized in the adopted energy or EPI principle. Thus the infimum of the Fisher measure implies the supremum of the average gradient of the wave function and hence its lowest degree of structure ("order"). This further implies the highest admissible degree of the wave-function indeterminacy ("disorder") marked by the supremum of the complementary measure of the quantum Shannon entropy:

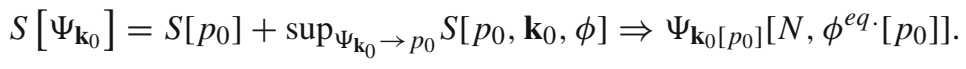

Therefore, the two quantum generalized measures of the information content of the complex wave-function in the Harriman-type construction are complementary in character: the ground-state density/energy constrained EPI of the lowest quantum Fisher information is synonymous with the related principle of the highest quantum Shannon entropy. This is again reminiscent of the complementary equilibrium criteria of 
the minimum energy and the maximum entropy in phenomenological thermodynamics [33].

\section{Phase descriptors of molecular fragments}

This phase approach to equilibria in molecules offers a new perspective on the promoted states of molecular fragments $\left\{\mathrm{M}_{\alpha}\right\}$, e.g., reactants $\left\{\mathrm{R}_{\alpha}\right\}$, $\mathrm{AIM}\left\{\mathrm{X}_{\alpha}\right\}$, etc., which determine the mutully exclusive pieces $\left\{\rho_{\alpha}=N_{\alpha} p_{\alpha}\right\}, N_{\alpha}=\int \rho_{\alpha}(\boldsymbol{r}) d \boldsymbol{r}$, of the system resultant density $\rho=N p$ in the isoelectronic molecular (M) or promolecular $\left(\mathrm{M}^{0}\right)$ systems as a whole:

$$
\begin{aligned}
& \rho(\boldsymbol{r})=\sum_{\alpha} \rho_{\alpha}(\boldsymbol{r})=\sum_{\alpha} N_{\alpha} p_{\alpha}(\boldsymbol{r})=N p(\boldsymbol{r}) \\
& p(\boldsymbol{r})=\sum_{\alpha}\left(N_{\alpha} / N\right) p_{\alpha}(\boldsymbol{r}) \equiv \sum_{\alpha} P_{\alpha} p_{\alpha}(\boldsymbol{r}), \\
& \sum_{\alpha} P_{\alpha}=\int p_{\alpha}(\boldsymbol{r}) d \boldsymbol{r}=1, \quad \sum_{\alpha} N_{\alpha}=N=\sum_{\alpha} N_{\alpha}^{0}=N^{0} .
\end{aligned}
$$

The (isoelectronic) promolecule corresponding to the given neutral system consists of its (molecularly) placed free constituent atoms. By linking the horizontal equilibrium phase to the system electron distribution one can explicitly specify the molecular/promolecular origins of such subsystems, thus distinguishing the isolated fragments, surrounded by an empty space, from their molecular/promolecular analogs. Indeed, the latter represent constituent parts of a larger (composed) system, being surrounded by their respective molecular/promolecular remainders. Thus, the internal equilibrium states of isolated species are fully determined by their own densities alone, while the phases of the external equilibria in molecular fragments are fixed by the density of a larger, composed system they belong to.

For example, the free (isolated, infinitely separated) atoms $\left\{\mathrm{X}_{\alpha}^{0}(\infty)\right\}$, exhibiting the atomic/ionic ground-state densities $\left\{\rho_{\alpha}^{0}=N_{\alpha}^{0} p_{\alpha}^{0}\right\}, N_{\alpha}^{0}=$ integer, and surrounded by an empty space are now characterized by the equilibrium phases $\left\{\varphi_{\alpha, e q .}^{0}(\infty)=\right.$ $\left.\phi_{e q .}\left[\boldsymbol{r} ; N_{\alpha}^{0}, p_{\alpha}^{0}\right]\right\}$, while their promolecular analogs of the same densities, i.e., the molecularly placed free-atoms, now correspond to the inter-fragment equalized promolecular phase $\left\{\varphi_{\alpha, e q}^{0} .\left(\mathrm{M}^{0}\right)=\phi_{e q} .\left[\boldsymbol{r} ; N^{0}, p^{0}\right]\right\}$ determined by the overall density or probability distribution of the whole composed system $\mathrm{M}^{0}$ :

$$
\begin{aligned}
& p^{0}(\boldsymbol{r})=\rho^{0}(\boldsymbol{r}) / N^{0}, \rho^{0}(\boldsymbol{r})=\sum_{\alpha} \rho_{\alpha}^{0}(\boldsymbol{r})=\sum_{\alpha} N_{\alpha}^{0} p_{\alpha}^{0}(\boldsymbol{r})=N^{0} p^{0}(\boldsymbol{r}) \quad \text { or } \\
& p^{0}(\boldsymbol{r})=\sum_{\alpha}\left(N_{\alpha}^{0} / N\right) p_{\alpha}^{0}(\boldsymbol{r}) \equiv \sum_{\alpha} P_{\alpha}^{0} p_{\alpha}^{0}(\boldsymbol{r}), \sum_{\alpha} P_{\alpha}^{0}=\int p_{\alpha}^{0}(\boldsymbol{r}) d \boldsymbol{r}=1 .
\end{aligned}
$$

The equilibrium states of molecular fragments, e.g., the bonded atoms $\left\{\mathrm{X}_{\alpha}\right\}$ corresponding to densities $\left\{\rho_{\alpha}(\boldsymbol{r})\right\}$, i.e., the AIM pieces of the molecular ground-state density $\rho(\boldsymbol{r})$, are similarly specified by the equilibrium phase of the whole molecule 
$\mathrm{M},\left\{\varphi_{\alpha, e q .}(\mathrm{M})=\phi_{e q .}\left[\boldsymbol{r} ; N, p_{0}\right]\right\}$, determined by the molecular ground-state probability distribution $p_{0}$, while the isolated AIM fragments, in an empty space, exhibit the subsystem phases $\left\{\varphi_{\alpha, e q .}(\infty)=\phi_{e q .}\left[\boldsymbol{r} ; N_{\alpha}, p_{\alpha}\right]\right\}$.

Therefore, the present approach provides the full specification of molecular fragments, including both the density/probability distribution of the fragment itself and its equilibrium phase identyfying the composed system from which this subsystem originates. The "frozen", molecularly placed free-atoms in the system promolecule, are thus phase-promoted compared to their isolated analogs. The promolecular density $\rho^{0}(\boldsymbol{r})$ thus induces the promolecular current in the molecularly placed free atom $\mathrm{X}_{\alpha}^{0}\left(\mathrm{M}^{0}\right)$,

$$
j_{\alpha}^{0}\left(\mathrm{M}^{0}\right)=\frac{\hbar}{m} p_{\alpha}^{0} \nabla \varphi_{\alpha, e q .}^{0}\left(\mathrm{M}^{0}\right)=-\frac{\hbar}{2 m}\left(\frac{p_{\alpha}^{0}}{p^{0}}\right) \nabla p^{0}
$$

which differs from the equilibrium current in the isolated atom $\mathrm{X}_{\alpha}^{0}(\infty)$,

$$
j_{\alpha}^{0}(\infty)=\frac{\hbar}{m} p_{\alpha}^{0} \nabla \phi_{\alpha, e q .}^{0}(\infty)=-\frac{\hbar}{2 m} \nabla p_{\alpha}^{0}
$$

It should be noticed that the ratio $p_{\alpha}^{0}(\boldsymbol{r}) / p^{0}(\boldsymbol{r})$ in Eq. (88) is related to the corresponding local Hirshfeld (H) "share" $d_{\alpha}^{\mathrm{H}}(\boldsymbol{r})$ in the "stockholder" partition [9-11,25] of the molecular electron density $\rho(\boldsymbol{r})$ into AIM pieces $\left\{\rho_{\alpha}^{\mathrm{H}}(\boldsymbol{r})=N_{\alpha}^{\mathrm{H}} p_{\alpha}^{\mathrm{H}}(\boldsymbol{r})\right\}, N_{\alpha}^{\mathrm{H}}=\int \rho_{\alpha}^{\mathrm{H}}(\boldsymbol{r}) d \boldsymbol{r}$,

$$
\rho_{\alpha}^{\mathrm{H}}=\left(\rho_{\alpha}^{0} / \rho^{0}\right) \rho \equiv d_{\alpha}^{\mathrm{H}} \rho, \quad d_{\alpha}^{\mathrm{H}}=P_{\alpha}^{0}\left(p_{\alpha}^{0} / p^{0}\right) .
$$

A similar current-distinction between the equilibrium state of the isolated AIM fragments $\left\{\mathrm{X}_{\alpha}(\infty)\right\}$ and the corresponding bonded atoms $\left\{\mathrm{X}_{\alpha}(\mathrm{M})\right\}$ in a molecule reads:

$$
\begin{aligned}
& j_{\alpha}(\infty)=\frac{\hbar}{m} p_{\alpha} \nabla \varphi_{\alpha, e q .}(\infty)=-\frac{\hbar}{2 m} \nabla p_{\alpha} \quad \text { and } \\
& j_{\alpha}(\mathbf{M})=\frac{\hbar}{m} p_{\alpha} \nabla \varphi_{\alpha, e q .}(\mathbf{M})=-\frac{\hbar}{2 m}\left(\frac{p_{\alpha}}{p}\right) \nabla p .
\end{aligned}
$$

In the stockholder partitioning [25], for which [see Eq. (90)]

$$
d_{\alpha}^{\mathrm{H}}=\rho_{\alpha}^{\mathrm{H}} / \rho=N_{\alpha}^{\mathrm{H}} p_{\alpha}^{\mathrm{H}} /(N p) \equiv P_{\alpha}^{\mathrm{H}}\left(p_{\alpha}^{\mathrm{H}} / p\right)=d_{\alpha}^{0} \equiv \rho_{\alpha}^{0} / \rho^{0}=P_{\alpha}^{0}\left(p_{\alpha}^{0} / p^{0}\right),
$$

the local ratio of the AIM and molecular probability distributions in Eq. (91) reads:

$$
\left(p_{\alpha}^{\mathrm{H}} / p\right)=\left(P_{\alpha}^{0} / P_{\alpha}^{\mathrm{H}}\right)\left(p_{\alpha}^{0} / p^{0}\right)=\left(N_{\alpha}^{0} / N_{\alpha}^{\mathrm{H}}\right)\left(p_{\alpha}^{0} / p^{0}\right)
$$

One also observes that the Hirshfeld division scheme can be interpreted as the universal (AIM-independent) enhancement of the free-atom densities:

$$
\rho_{\alpha}^{\mathrm{H}}=\rho_{\alpha}^{0}\left(\rho / \rho^{0}\right) \equiv \rho_{\alpha}^{0} w, \quad \alpha=\mathrm{A}, \mathrm{B}, \ldots,
$$




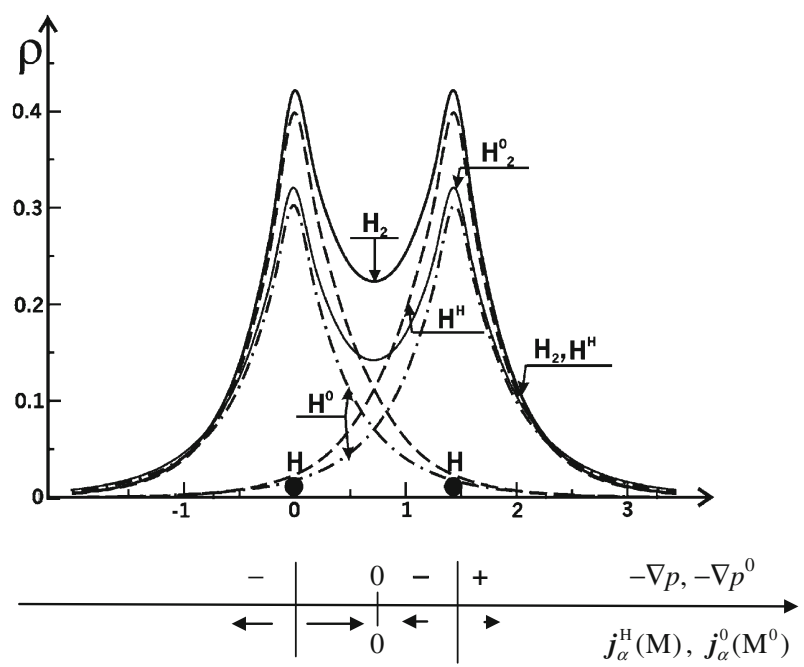

Fig. 1 A qualitative analysis of directions/magnitudes of the equilibrium AIM current $j_{\alpha}^{\mathrm{H}}(\mathrm{M})$ in the left hydrogen of $\mathrm{H}_{2}$ for the axial cut of the stockholder partition of the electron density in $\mathrm{H}_{2}$. The Hirshfeld electron densities of the bonded hydrogen atoms $\left(\mathrm{H}^{\mathrm{H}}\right)$ are obtained by the stockholder partition of the molecular density $\left(\mathrm{H}_{2}\right)$. The free-hydrogen densities $\left(\mathrm{H}^{0}\right)$ and the resulting electron density of the promolecule $\left(\mathrm{H}_{2}^{0}\right)$ are also shown for comparison. The density values and distances are in a.u. The zero cusps at nuclear positions are artifacts of the Gaussian basis set used in DFT calculations. The same patterns of directions/magnitudes characterize the equilibrium currents $j_{\alpha}^{0}\left(\mathrm{M}^{0}\right)$ of constituent atoms $\left\{\mathrm{X}^{0}\left(\mathrm{M}^{0}\right)\right\}$ in the promolecule $\mathrm{M}^{0}$

where the molecular enhancement factor

$$
w=\rho / \rho^{0}=p / p^{0}=\rho_{\alpha}^{\mathrm{H}} / \rho_{\alpha}^{0}=\left(N_{\alpha}^{\mathrm{H}} / N_{\alpha}^{0}\right)\left(p_{\alpha}^{\mathrm{H}} / p_{\alpha}^{0}\right) .
$$

The equilibrium states of isolated molecular fragments are thus distinguished from those of their bonded analogs by their phase/current descriptors. The bonded subsystems are characterized by the gradients of the molecular phase, related to molecular velocities of the probability fluid, while the free fragments exhibit the particle velocities of the subsystem in question. The change in the Hirshfeld AIM current due to the bond formation with its molecular environment reads:

$$
\begin{aligned}
\Delta j_{\alpha}^{\mathrm{H}}(\mathrm{M} ; \infty) & =j_{\alpha}^{\mathrm{H}}(\mathrm{M})-j_{\alpha}^{\mathrm{H}}(\infty)=-\frac{\hbar}{2 m}\left[p_{\alpha}^{\mathrm{H}}\left(\nabla \ln p-\nabla \ln p_{\alpha}^{\mathrm{H}}\right)\right] \\
& =\frac{\hbar}{2 m} p_{\alpha}^{\mathrm{H}} \nabla \ln \left(\frac{p_{\alpha}^{\mathrm{H}}}{p}\right)=\frac{\hbar}{2 m} p_{\alpha}^{\mathrm{H}} \nabla \ln \left(\frac{d_{\alpha}^{\mathrm{H}}}{P_{\alpha}^{\mathrm{H}}}\right) .
\end{aligned}
$$

Of interest also are displacements in the equilibrium currents of bonded atoms relative to their respective free analogs in the promolecular reference:

$$
\Delta j_{\alpha}^{\mathrm{H}}\left(\mathrm{M} ; \mathrm{M}^{0}\right)=j_{\alpha}^{\mathrm{H}}(\mathrm{M})-j_{\alpha}^{0}\left(\mathrm{M}^{0}\right)=\frac{\hbar}{2 m}\left(\frac{p_{\alpha}^{0}}{p^{0}} \nabla p^{0}-\frac{p_{\alpha}^{\mathrm{H}}}{p} \nabla p\right)
$$




$$
\begin{aligned}
& =\frac{\hbar}{2 m} \frac{p_{\alpha}^{\mathrm{H}}}{p}\left[\left(\frac{N_{\alpha}^{\mathrm{H}}}{N_{\alpha}^{0}}-w\right) \nabla p^{0}-p^{0} \nabla w\right] \\
& =\frac{\hbar}{2 m} \frac{d_{\alpha}^{\mathrm{H}}}{P_{\alpha}^{\mathrm{H}}}\left[\left(\frac{N_{\alpha}^{\mathrm{H}}}{N_{\alpha}^{0}}-w\right) \nabla p^{0}-p^{0} \nabla w\right] .
\end{aligned}
$$

As an illustration we have qualitatively examined in Fig. 1 the directions and relative magnitudes of the equilibrium probability currents in the Hirshfeld AIM $\left(\mathrm{H}^{\mathrm{H}}\right)$ in $\mathrm{H}_{2}$, implied by the gradient of the thermodynamic contribution, $-(1 / 2) \ln p$, of the molecular equilibrium phase. As shown in the figure, the direction of electron flow is determined by the negative gradient of the molecular density $\left(\mathrm{H}_{2}\right)$, while its resultant magnitude results from a subsequent weighting of this gradient with the Hirshfeld share factor, reflecting a local participation of the free atom density $\left(\mathrm{H}^{0}\right)$ in the promolecular distribution $\left(\mathrm{H}_{2}^{0}\right)$. Directions of local currents in the bonding region, between the two nuclei, are seen to concentrate electrons near the mid point of this prototype covalent chemical bond, while in the non-bonding regions they tend to expand the atomic distributions, effectively promoting a larger size of bonded atoms in their effective "valence" states. The bonding flows thus tend to enhance the known effects of the chemical bond, manifested by the polarization of the bonded atoms towards their molecular partners, while the non-bonding (promotional) "flows" act in a direction to restore the initial electron distribution of an isolated hydrogen, which is seen to be more "diffused" compared to the stockholder AIM. One also observes that the same pattern of directions/magnitudes of the probability currents describes the constituent freeatomic fragments of the system promolecule. In the bonding region these equilibrium flows are thus seen to "push" these atomic fragments towards their eventual equilibrium state in the molecule.

\section{Elements of non-equilibrium thermodynamic description}

For reasons of simplicity we again focus on one-electron systems, $N=1$, when particle density "variable" also reflects its probability distribution: $\rho(\boldsymbol{r})=p(\boldsymbol{r})$. This density and the current distribution $\boldsymbol{j}(\boldsymbol{r})$, which respectively reflect the modulus- and phase-parts of a general (complex) quantum state of Eq. (1), constitute two independent degrees-of-freedom of the system electronic structure.

The first "variable" $\rho(\boldsymbol{r})$ represents the local extensive parameter, since its value in the composite molecular system is the sum of its values in the molecular subsystems of interest. In the closed system the closure condition then requires

$$
\sum_{\alpha} \int \rho_{\alpha}(\boldsymbol{r}) d \boldsymbol{r}=\sum_{\alpha} N_{\alpha}=\int \rho(\boldsymbol{r}) d \boldsymbol{r}=N^{0}, \text { an integer constant. }
$$

Since this extensive parameter can be neither produced nor destroyed, the local change in electron density is solely due to the particle outflow, represented by the negative divergence of probability current $\boldsymbol{j}(\boldsymbol{r})$, thus giving rise to the source-less form of the continuity equation [Eqs. $(10,11)]$. 
The phase "variable" itself is not extensive in character and so is the phase-density $\pi=\phi^{2}$. In fact these two variables can be regarded as "thermodynamic" intensities, since their local values in the equilibrium state of molecular subsystems are equalized at the corresponding values of these phase descriptors of the system as a whole. Thus, the product of the given intensive phase parameter, e.g., $\nabla \phi$, with the appropriate density component, e.g., $\rho(\boldsymbol{r}) \nabla \phi(\boldsymbol{r})=p(\boldsymbol{r}) \nabla \phi(\boldsymbol{r})$ in Eq. (3), constitutes a bona-fide extensive parameter of general molecular states.

These two independent degrees of freedom are mutually coupled, evolving in time in accordance with the SE (7). Elsewhere $[16,17]$ an attempt has been made to derive the continuity equation for the phase-density aspect of the molecular electronic structure, with an appropriately defined phase-current, which in general quantum states was shown to exhibit a non-vanishing phase-source. Since these earlier identifications were non-unique, in this section we shall briefly reexamine these concepts in an attempt to provide a fully symmetric treatment of the density and phase aspects of molecular states. We shall also introduce the associated concept of the quantum entropy-current and derive the associated expression for the entropy-source in the associated continuity equation. Establishing these elementary concepts is vital for ultimately confronting the rates of non-equilibrium processes in molecular/reactive systems and providing their phenomenological description, in the spirit of the ordinary irreversible thermodynamics [33].

One first observes that the local currents $(\boldsymbol{j}, \boldsymbol{J})$, of the particle density and of the state phase-density $\pi$, respectively, are driven by the same particle velocity [see Eq. (13)],

$$
\boldsymbol{V}=(\hbar / m) \nabla \phi=[\hbar /(m p)] \operatorname{Im}\left[\varphi^{*} \nabla \varphi\right]
$$

This common speed of the probability and phase "fluids" reflects the associated currents-per-particle:

$$
\boldsymbol{V}=\boldsymbol{j} / p=(\hbar / m) \nabla \phi=[\hbar /(m p)] \operatorname{Im}\left[\varphi^{*} \nabla \varphi\right] \equiv \boldsymbol{J} / \pi
$$

The two currents thus exhibit the same direction as $\boldsymbol{V}$ and their magnitudes are interrelated:

$$
j / J=(p / \pi) .
$$

Since the probability current $j$ reflects the phase-gradient $\nabla \phi$, one would expect that the phase current $\boldsymbol{J}$ should be linked to the probability-gradient. These hints lead to the following, symmetrical treatment of the flows (fluxes) exhibited by these two independent degrees-of-freedom of molecular states, which ultimately reflect the independent real and imaginary parts of the system wave function:

$$
\begin{aligned}
& \boldsymbol{j}=(\hbar / m) p \nabla \phi=(\hbar / 2 m)(p / \phi) \nabla \pi \equiv \boldsymbol{j}_{p}, \\
& \boldsymbol{J}=(\hbar / m) \pi \nabla R=(\hbar / 2 m)(\pi / R) \nabla p \equiv \boldsymbol{J}_{\pi}
\end{aligned}
$$


From the SE (7) and its Hermitian conjugate one obtains the following time derivatives of the distribution amplitudes [16,17],

$$
\begin{aligned}
\partial R / \partial t & =-(\hbar / m)[\nabla R \cdot \nabla \phi+(R / 2) \Delta \phi], \\
\partial \phi / \partial t & =(\hbar / 2 m)]\left[(\nabla \phi)^{2}-R^{-1} \Delta R\right]-v / \hbar,
\end{aligned}
$$

and hence also the associated derivatives of the distributions themselves:

$$
\partial p / \partial t=2 R(\partial R / \partial t) \text { and } \partial \pi / \partial t=2 \phi(\partial \phi / \partial t)
$$

We shall now apply these time derivatives in the associated continuity equations. One directly verifies that the probability derivative gives rise to Eq. (10), where the divergence of the probability-current

$$
\nabla \cdot \boldsymbol{j}=(\hbar / m)[\nabla p \cdot \nabla \phi+p \Delta \phi]=(\hbar / m)\left[2 R \nabla R \cdot \nabla \phi+R^{2} \Delta \phi\right] .
$$

For the divergence of the phase-current one similarly obtains the complementary expression:

$$
\nabla \cdot \boldsymbol{J}=(\hbar / m)[\nabla \pi \cdot \nabla R+\pi \Delta R]=(\hbar / m)\left[2 \phi \nabla \phi \cdot \nabla R+\phi^{2} \Delta R\right]
$$

Combining Eqs. (104), (105) and (107) then gives the following source of the phase density in the continuity equation for this component:

$$
\begin{aligned}
d \pi / d t & \equiv \dot{\pi}=\sigma_{\pi}=\partial \pi / \partial t+\nabla \cdot \boldsymbol{J} \\
& =\phi\left\{(\hbar / m)\left[(\nabla \phi+2 \nabla R) \cdot \nabla \phi+\left(\phi-R^{-1}\right) \Delta R\right]-2 v / \hbar\right\} .
\end{aligned}
$$

Next, let us address the related problem of the quantum-entropy source. Following the standard approach in ordinary irreversible thermodynamics [33], we first identify the quantum-entropy conjugates of the state two local state variables $p$ and $\pi$,

$$
\begin{aligned}
& F_{p}(\boldsymbol{r})=\delta S[\varphi] / \delta p(\boldsymbol{r})=\frac{\partial \mathscr{S}(\boldsymbol{r})}{\partial p(\boldsymbol{r})}=-\ln p(\boldsymbol{r})-2 \pi(\boldsymbol{r})^{1 / 2}-1, \\
& F_{\pi}(\boldsymbol{r})=\delta S[\varphi] / \delta \pi(\boldsymbol{r})=\frac{\partial \mathscr{S}(\boldsymbol{r})}{\partial \pi(\boldsymbol{r})}=-p(\boldsymbol{r}) / \pi(\boldsymbol{r})^{1 / 2}
\end{aligned}
$$

They determine the associated affinities, "thermodynamic" forces, defined as gradients of these intensities:

$$
\begin{aligned}
& \mathscr{F}_{p}=\nabla F_{p}=-\left[p^{-1} \nabla p+2 \nabla\left(\pi^{1 / 2}\right)\right]=-2\left[R^{-1} \nabla R+\nabla \phi\right], \\
& \mathscr{F}_{\pi}=\nabla F_{\pi}=\pi^{-1}\left[p \nabla\left(\pi^{1 / 2}\right)-\pi^{1 / 2} \nabla p\right]=2(R / \phi)[(R / \phi) \nabla \phi-2 \nabla R] .
\end{aligned}
$$

One also introduces the current density of the local quantum entropy,

$$
\boldsymbol{J}_{\mathscr{S}}(\boldsymbol{r})=F_{p}(\boldsymbol{r}) \boldsymbol{j}_{p}(\boldsymbol{r})+F_{\pi}(\boldsymbol{r}) \boldsymbol{J}_{\pi}(\boldsymbol{r}),
$$


determining in the entropy continuity equation the rate of increase of the entropy density within the infinitesimal region in question:

$$
d \mathscr{S}(\boldsymbol{r}) / d t \equiv \dot{\mathscr{S}}(\boldsymbol{r})=\sigma_{\mathscr{S}}(\boldsymbol{r})=\partial \mathscr{S}(\boldsymbol{r}) / \partial t+\nabla \cdot \boldsymbol{J}_{\mathscr{S}}(\boldsymbol{r})
$$

Its first term is suggested by the entropy differential

$$
\begin{aligned}
d S[\varphi]=d S[p, \pi] & =\int \frac{\partial \mathscr{S}(\boldsymbol{r})}{\partial p(\boldsymbol{r})} d p(\boldsymbol{r}) d \boldsymbol{r}+\int \frac{\partial \mathscr{S}(\boldsymbol{r})}{\partial \pi(\boldsymbol{r})} d \pi(\boldsymbol{r}) d \boldsymbol{r} \\
& =\int\left[F_{p}(\boldsymbol{r}) d p(\boldsymbol{r})+F_{\pi}(\boldsymbol{r}) d \pi(\boldsymbol{r})\right] d \boldsymbol{r},
\end{aligned}
$$

and determined by Eqs. (103)-(105):

$$
\partial \mathscr{S}(\boldsymbol{r}) / \partial t=F_{p}(\boldsymbol{r}) \frac{\partial p(\boldsymbol{r})}{\partial t}+F_{\pi}(\boldsymbol{r}) \frac{\partial \pi(\boldsymbol{r})}{\partial t} .
$$

The divergence of Eq. (111),

$$
\nabla \cdot \boldsymbol{J}_{\mathscr{S}}=\left(\nabla F_{p}\right) \cdot \boldsymbol{j}_{p}+F_{p} \nabla \cdot \boldsymbol{j}_{p}+\left(\nabla F_{\pi}\right) \cdot \boldsymbol{J}_{\pi}+F_{\pi} \nabla \cdot \boldsymbol{J}_{\pi},
$$

combined with the probability and phase continuity relations [Eqs. (11) and (108)] finally gives the following, thermodynamic-like expression for the rate of the local production of quantum entropy:

$$
\sigma_{\mathscr{S}}(\boldsymbol{r})=\mathscr{F}_{p}(\boldsymbol{r}) \cdot \boldsymbol{j}_{p}(\boldsymbol{r})+\mathscr{F}_{\pi}(\boldsymbol{r}) \cdot \boldsymbol{J}_{\pi}(\boldsymbol{r})+F_{\pi}(\boldsymbol{r}) \sigma_{\pi}(\boldsymbol{r})
$$

Therefore, the entropy production identically vanishes only in the stationary quantum state, e.g., the exact ground state of a molecule, for $\phi=0$, i.e., $\sigma_{\pi}=0$, when both affinities vanish: $\mathscr{F}_{p}=\mathscr{F}_{\pi}=\boldsymbol{0}$. However, contrary to the ordinary irreversible thermodynamics [33], these zero affinities do not lead to the vanishing quantum entropy source in general quantum states, for which both $F_{\pi}$ and $\sigma_{\pi}$ assume nonzero values.

\section{Conclusion}

The quantum extension of the classical entropy/information concepts accounts for the information content due to both the density and probability current (phase) distributions in the complex electronic states. In this generalized framework the non-classical (quantum) information contributions complement the classical Fisher and Shannon information measures, functionals of the particle probability distribution alone, in the resultant entropic descriptors, which extract the full information content of the complex probability amplitudes (wave functions) of the quantum mechanical description.

The entropic variational principles for determining the molecular vertical and horizontal equilibria, for the fixed and unconstrained particle density, respectively, have 
been investigated. These phase-equilibria of atomic and molecular systems correspond to the extrema of quantum information measures. The vertical principle identifies the orthogonality phase, giving rise to the vanishing overall current, as in the stationary ground-state, while the horizontal rule gives rise to the IT-optimum phase related to the logarithm of the system electron probability distribution. The latter generates a non-vanishing probability current responsible for the finite non-classical entropy/information contributions.

The double (density and phase/current) description of molecular fragments, e.g., AIM, provides the full specification of the equilibrium states of such subsystems. The equilibrium quantum information descriptors then reflect both the molecular origins of AIM and the environment of isolated atoms in the system promolecule. Implications of the phase-equilibria for the state of bonded Hirshfeld atoms in $\mathrm{H}_{2}$ have been examined in some detail. We have argued, that such complete specification of the subsystem equilibria requires the entropic representation of IT. Indeed, the EPI principles of the latter determine the fragment equilibrium phase, which reflects its environment in a larger, composite system.

Finally, the elements of an irreversible-thermodynamical description of molecular states have been introduced. They include the state-parameters reflecting the particle probability (wave-function modulus) and the particle current (wave-function phase), their associated quantum-entropy conjugates ("intensities") and affinities (gradients of "intensities"). The phase-continuity equation has been derived, the quantum entropy current has been introduced and the associated entropy continuity equation has identified the local entropy production in general quantum states. The latter has been shown to identically vanish only for the vanishing affinities in the stationary quantum state, for the sharply specified electronic energy and vanishing spatial phase.

\section{References}

1. R.A. Fisher, Proc. Camb. Philos. Soc. 22, 700 (1925)

2. B.R. Frieden, Physics from the Fisher Information-A Unification, 2nd edn. (Cambridge University Press, Cambridge, 2004)

3. C.E. Shannon, Bell Syst. Tech. J. 27, 379, 623 (1948)

4. C.E. Shannon, W. Weaver, The Mathematical Theory of Communication (University of Illinois, Urbana, 1949)

5. S. Kullback, R.A. Leibler, Ann. Math. Stat. 22, 79 (1951)

6. S. Kullback, Information Theory and Statistics (Wiley, New York, 1959)

7. N. Abramson, Information Theory and Coding (McGraw-Hill, New York, 1963)

8. P.E. Pfeifer, Concepts of Probability Theory, 2nd edn. (Dover, New York, 1978)

9. R.F. Nalewajski, R.G. Parr, Proc. Natl. Acad. Sci. USA 97, 8879 (2000)

10. R.F. Nalewajski, R.G. Parr, J. Phys. Chem. A 105, 7391 (2001)

11. R.F. Nalewajski, Information Theory of Molecular Systems (Elsevier, Amsterdam, 2006)

12. R.F. Nalewajski, Information Origins of the Chemical Bond (Nova, New York, 2010)

13. R.F. Nalewajski, Perspectives in Electronic Structure Theory (Springer, Heidelberg, 2012)

14. R.F. Nalewajski, in Frontiers, in Modern Theoretical Chemistry: Concepts and Methods (Dedicated to B. M. Deb), ed. by P.K. Chattaraj, S.K. Ghosh (Taylor \& Francis/CRC, London, 2013), pp. 143-180

15. R.F. Nalewajski, Struct. Bond. 149, 51 (2012)

16. R.F. Nalewajski, J. Math. Chem. 51, 297 (2013)

17. R. F. Nalewajski, Found. Chem. 16, 27 (2014); Ibid., J. Math.Chem. 52, 588 (2014)

18. R.F. Nalewajski, J. Math. Chem. 51, 369 (2013)

19. R.F. Nalewajski, Ann. Phys. Leipzig 525, 256 (2013) 
20. R.G. Parr, P.W. Ayers, R.F. Nalewajski, J. Phys. Chem. A 109, 3957 (2005)

21. R.F. Nalewajski, A.M. Köster, S. Escalante, J. Phys. Chem. A 109, 10038 (2005)

22. R.F. Nalewajski, J. Math. Chem. 47, 667 (2010)

23. R.F. Nalewajski, P. de Silva, J. Mrozek, J. Mol. Struct: THEOCHEM 954, 57 (2010)

24. R.F. Nalewajski, J. Phys. Chem. A 104, 11940 (2000)

25. F.L. Hirshfeld, Theor. Chim. Acta (Berl.) 44, 129 (1977)

26. R.F. Nalewajski, Int. J. Quantum Chem. 108, 2230 (2008)

27. A.D. Becke, K.E. Edgecombe, J. Chem. Phys. 92, 5397 (1990)

28. B. Silvi, A. Savin, Nature 371, 683 (1994)

29. A. Savin, R. Nesper, S. Wengert, T.F. Fässler, Angew. Chem. Int. Ed. Engl. 36, 1808 (1997)

30. R.F. Nalewajski, J. Phys. Chem A 107, 3792 (2003)

31. R.F. Nalewajski, Mol. Phys. 104, 255 (2006)

32. R.F. Nalewajski, Ann. Phys. Leipzig 13, 201 (2004)

33. H.B. Callen, Thermodynamics: An Introduction to the Physical Theories of the Equilibrium Thermostatics and Irreversible Thermodynamics (Wiley, New York, 1960)

34. C.F. von Weizsäcker, Z. Phys. 96, 431 (1935)

35. P. Hohenberg, W. Kohn, Phys. Rev. 136B, 864 (1964)

36. W. Kohn, L.J. Sham, Phys. Rev. 140A, 1133 (1965)

37. J.E. Harriman, Phys. Rev. A 24, 680 (1981)

38. G. Zumbach, K. Maschke, Phys. Rev. A 28, 544 (1983); erratum. Phys. Rev. A 29, 1585 (1984)

39. R.F. Nalewajski, J. Math. Chem. 51, 369 (2013)

40. M. Levy, Proc. Natl. Acad. Sci. USA 76, 6062 (1979)

41. W. Macke, Ann. Phys. Leipzig 17, 1 (1955)

42. T.L. Gilbert, Phys. Rev. B 12, 2111 (1975)

43. R.G. Parr, M.-B. Chen, Proc. Natl. Acad. Sci. USA 78, 1323 (1981)

44. D.R. Hartree, Proc. Camb. Philos. Soc. 24, 89 (1928)

45. V.A. Fock, Z. Phys. 61, 126 (1930) 